\title{
Interrogation of Enhancer Function by Enhanced CRISPR Epigenetic Editing
}

Kailong $\mathrm{Li}^{1,2,4}$, Yuxuan $\mathrm{Liu}^{1,2,4}$, Hui Cao ${ }^{1,2}$, Yuannyu Zhang ${ }^{1,2}$, Zhimin $\mathrm{Gu}^{1,2}$, Xin $\mathrm{Liu}^{1,2}$, Andy Yu ${ }^{1,3}$, Pranita Kaphle ${ }^{1,2}$, Kathryn E. Dickerson ${ }^{1,2}$, Min $\mathrm{Ni}^{1,2}$, Jian $\mathrm{Xu}^{1,2, *}$

${ }^{1}$ Children's Medical Center Research Institute, University of Texas Southwestern Medical Center, Dallas, TX 75390, USA

${ }^{2}$ Department of Pediatrics, Harold C. Simmons Comprehensive Cancer Center, and Hamon Center for Regenerative Science and Medicine, University of Texas Southwestern Medical Center, Dallas, TX 75390, USA

${ }^{3}$ SURF-Stem Cell Program, University of Texas Southwestern Medical Center, Dallas, TX 75390, USA

${ }^{4}$ Co-first author

*Correspondence: jian.xu@utsouthwestern.edu (J.X.)

Mailing address:

6000 Harry Hines Blvd.

C. Kern Wildenthal Research Building - NL12.138B

UT Southwestern Medical Center

Dallas, Texas 75390-8502

Phone: 214-648-6125

Running Title: Enhancer Interrogation by Enhanced Epigenetic Editing

Keywords: Enhancer, Chromatin, Epigenetics, CRISPR/Cas9, Hematopoiesis 


\section{ABSTRACT}

Tissue-specific gene expression requires coordinated control of gene-proximal and -distal cisregulatory elements (CREs), yet functional analysis of gene-distal CREs such as enhancers remains challenging. Here we describe enhanced CRISPR/dCas9-based epigenetic editing systems, enCRISPRa and enCRISPRi, for multiplexed analysis of enhancer function in situ and in vivo. Using dual effectors capable of re-writing enhancer-associated chromatin modifications, we show that enCRISPRa and enCRISPRi modulate gene transcription by remodeling local epigenetic landscapes at sgRNA-targeted enhancers and associated genes. Comparing with existing methods, the new systems display more robust perturbation of enhancer activity and gene transcription with minimal off-targets. Allele-specific targeting of enCRISPRa to oncogenic TAL1 super-enhancer modulates TAL1 expression and cancer progression in xenotransplants. Multiplexed perturbations of lineage-specific enhancers using an enCRISPRi knock-in mouse establish in vivo evidence for lineage-restricted essentiality of developmental enhancers during hematopoietic lineage specification. Hence, enhanced CRSIPR epigenetic editing provides opportunities for interrogating enhancer function in native biological contexts. 


\section{INTRODUCTION}

Mammalian gene expression requires precisely regulated gene-proximal promoters and genedistal cis-regulatory elements (CREs) such as transcriptional enhancers. Systematic annotation of human epigenomes has identified millions of putative CREs using correlative features such as chromatin accessibility and histone modifications ${ }^{1-4}$; however, the in vivo functions of the vast majority of these elements within their native chromatin remain unknown. This is in part because existing technologies often measure enhancer activity in heterologous assays without native chromatin, and because findings from these assays have not been causally connected with specific target genes or cellular functions during development.

Enhancers are cis-regulatory DNA sequences that are bound and regulated by transcription factors (TFs) and chromatin regulators in a highly tissue-specific manner. Putative enhancers are operationally identified using epigenetic signatures including chromatin accessibility (DNase I hypersensitivity or ATAC-seq) and histone marks (H3K4me1/2 and H3K27ac) ${ }^{5-7}$. Unlike geneproximal promoters, enhancers can regulate gene transcription over long distances in an orientation-independent and cell-type-specific manner ${ }^{8}$. As such, fundamental challenges have limited the application of existing technologies in functional analysis of a specific enhancer in a mammalian genome. Reporter assays have historically been used to examine enhancer activity in heterologous cell models ${ }^{8}$. When combined with high-throughput sequencing, massively parallel reporter assays allow for quantitative analysis of the transcriptional activity of thousands of enhancers in particular cell types ${ }^{9,10}$. Together with transgenics, in vivo enhancer reporter assays enable evaluation of enhancer function during mammalian development ${ }^{11}$. These are powerful approaches for assaying TF-mediated transcriptional activity at enhancer DNA sequences, but they have some important limitations including the lack of local chromatin contexts and epigenetic features in heterologous assays, the use of a general promoter such as SV40 rather than the enhancer's endogenous promoter, the inability to identify the target genes of enhancers, and the inadequacy to model combinatorial regulation by multiple enhancers at native chromatin. Additionally, conventional gene targeting or genome editing approaches have been utilized to knockout (KO) or mutate specific enhancers in cell lines or animal models ${ }^{12,13}$; however, they require genetic engineering which remains low-throughput and laborious. Furthermore, high-resolution saturating screens of cis-regulatory elements rely on loss-offunction and do not permit gain-of-function analyses ${ }^{14-16}$.

Recently, major advances have been made in the modulation of endogenous gene expression by repurposing the CRISPR/Cas9 system ${ }^{17-29}$. By coupling the deactivated Cas9 (dCas9) to various activator (e.g. VP64 ${ }^{18-20}$, p300 ${ }^{22,28}$, and SAM ${ }^{21}$ ) or repressor (e.g. KRAB ${ }^{18, ~ 19, ~}{ }^{23,}{ }^{28}$, LSD1 ${ }^{29}$, and DNMT3A/3L ${ }^{26}$ ) domains, transcriptional perturbation of specific genes were achieved. While the most commonly used dCas9 activator or repressor complexes such as dCas9-VP64, dCas9-SAM or dCas9-KRAB can effectively modulate transcription when tethered to gene-proximal promoters, the effect declines rapidly when its target region moves away from 
proximal promoter sequences ${ }^{18-21}$. This is likely because VP64 or KRAB preferentially interferes with the basal transcription initiation and/or elongation apparatus operating at gene promoters ${ }^{29}$, 30. Since distal CREs such as enhancers may not rely on the basal transcription apparatus, these methods were ineffective and variable in modulating enhancer activity ${ }^{18-21}$.

Here we develop enhanced CRISPR epigenetic editing systems, enCRISPRa and enCRISPRi, to interrogate enhancer function using dCas9 with dual effectors that specifically modulate epigenetic modifications at enhancers. Using the human $\beta$-globin locus control region (LCR), oncogenic TAL1 super-enhancer (SE), and hematopoietic lineage-specific enhancers as examples, we show that enCRISPRa and enCRISPRi effectively modulate enhancer function in vitro, in xenografts and in vivo. Enhanced CRISPR epigenetic editing leads to locus-specific epigenetic reprogramming and interference with TF binding. Single or multiplexed in vivo enhancer perturbations using an enCRISPRi mouse model reveal lineage-specific requirements of developmental enhancers during hematopoiesis. Hence, the enhanced CRISPR epigenetic editing systems provide opportunities for functional interrogation of enhancers and other CREs in development and disease.

\section{RESULTS}

\section{Development of an enCRISPRa System for Enhancer Activation}

To assess the functional role of gene-distal enhancers, we devised the enhanced dCas9-based epigenetic perturbation systems for targeted modulation of enhancer activity in situ and in vivo. Specifically, we employed the structure-guided sgRNA design by adding two MS2 hairpins ${ }^{21}$, which is recognized by the MCP RNA-binding proteins ${ }^{31}$. For enhancer activation (enCRISPRa; Fig. 1a), we fused dCas9 with the core domain of histone acetyltransferase p300, which catalyzes H3-Lys27 acetylation (H3K27ac) ${ }^{32}$, together with the MS2-sgRNA sequence to recruit the MCP-VP64 activator domains. Since H3K27ac is the hallmark of active enhancers ${ }^{33,34}$, by doxycycline (Dox)-inducible expression of dCas9-p300, sgRNA-MS2 and MCP-VP64, we engineered an enhancer-targeting dual-activator system (Fig. 1a). As a proof-of-principle test of enCRISPRa in modulating CRE activity, we targeted enCRISPRa to several known enhancers or promoters including the MYOD enhancer, the ILIRN and OCT4 promoters ${ }^{22}$ in HEK293T cells (Fig. 1b). Comparing with existing next-generation dCas9-based activation methods such as dxCas9-VPR ${ }^{24}$, SunTag ${ }^{19}$ and SAM ${ }^{21}$, enCRISPRa showed comparable potency on gene activation when targeted to the ILIRN and OCT4 promoters (Fig. 1b). More importantly, enCRISPRa displayed significantly more robust activation of gene transcription compared to other dCas9 activators when targeted to the MYOD enhancer (Fig. 1b).

We next focused on the well-established HS2 enhancer at the human $\beta$-globin locus ${ }^{35}$ (Fig. 1c). The $\beta$-globin locus contains five $\beta$-like globin genes ( $H B E 1, H B G 1, H B G 2, H B D$ and $H B B$ ) that are developmentally regulated by a shared upstream enhancer cluster or locus control region 
(LCR) ${ }^{36}$. The $\beta$-globin LCR consists of five discrete enhancers (HS1 to HS5) in which HS2 functions as an erythroid-specific enhancer in transgenic assays ${ }^{36}$, and provides a paradigm for studying tissue-specific and developmentally regulated gene transcription. We engineered HEK293T cells with Dox-inducible expression of dCas9-p300 or other dCas9 activators ${ }^{19,} 21,24$. Upon co-expression of a single HS2-targeting sgRNA, we measured mRNA expression of $\beta$ globin genes and compared with existing dCas9 activators. Notably, enCRISPRa-mediated HS2 enhancer activation led to 13.0 to 40.6 -fold increases in expression of $\beta$-globin genes $H B E 1$, $H B G 1 / 2$ and $H B B$ relative to the non-transduced controls, which are significantly higher than other dCas9 activation methods (Fig. 1c). Although different dCas9 activators were noted to display variable potencies in gene activation in previous studies ${ }^{21,37-39}$, the observed differences could be affected by different cellular contexts, particular target genes, position of sgRNAs (e.g. promoter vs enhancer), transfection conditions, and time to analyze gene expression. Thus, it is important to note that our analyses were performed in the same cell lines using the same sgRNA and transfection protocol, which enabled us to compare the efficacy of different dCas9 activators on enhancer perturbation side-by-side. Moreover, analysis of dCas9 chromatin occupancy in cells co-expressing enCRISPRa and HS2-specific sgRNA (sgHS2) revealed highly reproducible binding at the targeted HS2 enhancer by independent ChIP-seq experiments (Fig. 1d; Table S1). By comparing dCas9 binding in cells expressing sgHS2 or nontargeting sgRNA (sgGal4), we observed highly specific enrichment of dCas9 at HS2 with no additional significant binding at the genome scale (Fig. 1d).

\section{Development of an enCRISPRi System for Enhancer Repression}

We next devised an enhanced epigenetic editing system for targeted enhancer inhibition (enCRISPRi-LK; Fig. 2a). Specifically, we fused dCas9 with the lysine-specific demethylase LSD1 (or KDM1A), which catalyzes the removal of enhancer-associated H3-Lys4 mono- and dimethylation (H3K4me1/2) ${ }^{40}$, together with MS2-sgRNA to recruit the MCP-KRAB repressor domains (Fig. 2a). As a proof-of-principle test, we targeted enCRISPRi to the HS2 enhancer in K562 erythroleukemia cells that highly express $\beta$-globin genes ${ }^{35}$. We achieved 3.4 to 13.7-fold repression of $\beta$-globin genes (HBE1, $H B G 1 / 2$ and $H B B$ ) by 4 independent sgRNAs (sgHS2-1 to $\mathrm{sgHS} 2-4$ ) relative to non-targeting sgGal4 (Fig. 2b). We further engineered the second version of enCRISPRi using dCas9-KRAB + MCP-LSD1 (enCRISPRi-KL) combination (Fig. 2a). Targeting of either enCRISPRi complex to the HS2 enhancer by 4 independent sgRNAs achieved comparable and significant repression of $\beta$-globin genes (Fig. $2 b$ ). Comparing with the single effector dCas9-KRAB (K) or dCas9-LSD1 (L) complex, the dual repressor-containing enCRISPRi displayed markedly stronger gene repression (Fig. 2b,c). In addition, enCRISPRi resulted in minimal changes (except $\beta$-globin genes) in global transcriptomics by RNA-seq (Fig. 2c). Moreover, ChIP-seq analyses revealed significant enrichment of dCas9 binding at the targeted HS2 enhancer with minimal off-targets by independent replicate experiments and/or comparing to the non-targeting sgGal4 control (Fig. 2d), indicating the locus-specific modulation of target gene transcription. 
We further explored the effectiveness of enCRISPRi by targeting to single or multiple enhancers at the $\beta$-globin LCR enhancer cluster (Fig. 2e). We focused on the enCRISPRi (LK) version given similar effects on gene repression when targeting enCRISPRi LK and KL to the HS2 enhancer (Fig. 2a-c). To this end, we designed sgRNAs for individual LCR enhancers (sgHS1 to sgHS5) and $H B G 1 / 2$ promoters (sgHBG). The $\beta$-globin locus is flanked by two CTCF-associated insulator elements at $\mathrm{HS} 5$ and $3^{\prime} \mathrm{HS} 1^{36}$. As important negative controls, we designed sgRNAs targeting DNA sequences $2 \sim 4 \mathrm{~kb}$ outside of the $\beta$-globin insulators (sgCTCF1 and sgCTCF2), outside of the $\beta$-globin locus-containing topologically associated domain (TAD) (sgTAD1 and sgTAD2), or at a different chromosome (sgCtrl; chr2:211,337,408-211,337,427) (Fig. 2e; Table S2). Upon stable co-expression of individual target-specific or control sgRNAs with enCRISPRi in $\mathrm{K} 562$ cells, we observed that sgHS2 resulted in more significant repression of all $\beta$-globin genes HBE1, HBG1/2 and $H B B$ (4.7 to 11.8 -fold, $P<0.001$ relative to sgGal4) compared to other LCR enhancers (Fig. 2f), consistent with the prominent role of the HS2 enhancer for LCR function ${ }^{35,36}$. Further, co-expression of all five sgRNAs targeting LCR enhancers (sgHS-all) did not further repress $\beta$-globin genes (Fig. 2e,f), suggesting that enCRISPRi was effective when targeted to a single enhancer by a single sgRNA. Notably, none of the control sgRNAs including two sgRNAs flanking $\beta$-globin insulators (sgCTCF1 and sgCTCF2) affected $\beta$-globin expression (Fig. 2e,f).

When targeted to gene-proximal promoters, dCas9-based epigenetic modulation may block TF binding and/or interfere with the formation of transcription initiation or elongation complexes ${ }^{17-19}$. By contrast, enhancer repression requires the interference with the function of specific enhancer-regulating TFs, chromatin regulators and their combinatorial activities. We reasoned that targeting enCRISPRi to the proximity of enhancer center may achieve maximal effects compared to enhancer distal sequences. To this end, we compared the efficacies of enCRISPRi by designing sgRNAs targeting the DNA sequences at the DNase I hypersensitivity (DHS) peak summit at HS2 (sgHS2), or the sequences located $0.5 \mathrm{~kb}$ or $2.5 \mathrm{~kb}$ upstream or downstream of the HS2 enhancer (sgHS2-2.5k, sgHS2-0.5k, sgHS2+0.5k and sgHS2+2.5k; Fig. 2e), respectively. We achieved the most significant gene repression when targeting enCRISPRi to enhancer DHS peak summit (3.6 to 9.1-fold, $P<0.001$ relative to sgGal4), and progressively decreased effects with increasing distances from enhancer center (Fig. 2f). These data emphasize the importance of targeting dCas9-based epigenetic editing complexes to the most accessible regions at enhancers for the maximal transcriptional perturbation.

Together, these results not only establish the improved epigenetic editing systems for enhancer perturbation, but also demonstrate that independent activators or repressors cooperate to modulate locus-specific gene transcription. It is important to note that, although the constituent components of enCRISPRa (p300 and VP64) and enCRISPRi (LSD1 and KRAB) have been tested for transcriptional modulation of promoter and/or enhancer activity by fusing to dCas9 individually $18,22,41$, the combinatorial effects on local epigenetic landscapes and gene transcription have not been examined previously. Therefore, the enCRISPRa and enCRISPRi 
systems that we describe here represent the first attempt to combine both p300-VP64 and LSD1-KRAB in a single dCas9 complex for targeted modulation of enhancer function, respectively.

\section{Locus-Specific Epigenetic Editing by enCRISPRi}

To determine the impact of enCRISPRi on epigenetic landscapes, we performed ChIP-seq analysis of dCas9, the enhancer-associated active histone marks (H3K4me1, H3K4me2 and $\mathrm{H} 3 \mathrm{~K} 27 \mathrm{ac}$ ), the repressive chromatin-associated $\mathrm{H} 3 \mathrm{~K} 9 \mathrm{me}$, the hematopoietic lineage master TFs (GATA1 and TAL1), and CTCF. We also compared cells expressing non-targeting sgGal4 (control or C; Fig. 3), or sgHS2 with the single effector dCas9-KRAB (K), dCas9-LSD1 (L) or the dual effector enCRISPRi (LK or KL) (two replicate experiments for each ChIP-seq, total 80 independent ChIP-seq experiments; Figs. 3, S1, S2 and S3; Table S1).

Comparing to control (C), dCas9-LSD1 (L) or dCas9-KRAB (K), the dual repressor enCRISPRi $\mathrm{LK}$ and $\mathrm{KL}$ resulted in more apparent loss of enhancer-associated activating histone marks H3K4me1 and H3K27ac at the targeted HS2 enhancer (Figs. 3, S1 and S3a-d). Both enCRISPRi LK and KL significantly increased the levels of the repressive histone mark H3K9me3 at the targeted HS2 enhancer but not the $\beta$-globin promoters (Figs. 3, S1 and S3e). Of note, enCRISPRi also led to marked loss of H3K4me2 and H3K27ac at the $\beta$-globin geneproximal promoters and gene bodies (Figs. 3 and S1), consistent with their transcriptional downregulation (Fig. 2b,c). These results suggest that enCRISPRi-mediated enhancer repression causes epigenetic changes at both targeted enhancers and associated gene promoters likely through enhancer-promoter interactions ${ }^{42}$. It is also important to note that, while dCas9-KRAB (K) led to significantly increased H3K9me3 at the targeted HS2 enhancer, dCas9-LSD1 (L) had no effect on H3K9me3 but instead decreased H3K4me1/2 (Fig. 3). These results are consistent with the roles of KRAB in promoting the formation of H3K9me3-mediated heterochromatin ${ }^{30}$ and LSD1 in the removal of H3K4me1/2 marks ${ }^{40}$. Further, enCRISPRi (LK or $\mathrm{KL}$ ) led to concurrent and more significant increases in H3K9me3 and decreases in H3K4me1/2 marks compared to dCas9-KRAB or dCas9-LSD1 alone (Figs. 3, S1 and S3b-e), illustrating the cooperative activity between two distinct repressor proteins. No significant enrichment of $\mathrm{H} 3 \mathrm{~K} 27 \mathrm{me} 3$, the repressive histone marks catalyzed by Polycomb proteins ${ }^{43}$, was detected at the $\beta$-globin gene cluster in K562 cells with or without dCas9-KRAB, dCas9-LSD1 or enCRISPRi (data not shown).

These data demonstrate that KRAB and LSD1 were capable of modulating epigenetic modifications by depositing $\mathrm{H} 3 \mathrm{~K} 9 \mathrm{me} 3$ and removing $\mathrm{H} 3 \mathrm{~K} 4 \mathrm{me} 1 / 2$, respectively, whereas the dual effector enCRISPRi led to more profound epigenetic changes, likely due to cooperation between distinct repressor domains. Finally, while dCas9-KRAB or dCas9-LSD1 alone slightly or modestly affected the binding of GATA1 and TAL1, the key hematopoietic TFs required for the HS2 enhancer function ${ }^{13,35,36}$, enCRISPRi led to further loss of GATA1 and TAL1 binding at 
the targeted HS2 enhancer (Figs. 3, S2 and S3f,g). No significant effect on CTCF binding to the flanking insulators (HS5 and 3'HS1) was observed (Figs. 3, S2 and S3h), suggesting that enCRISPRi results in locus-wide epigenetic changes that are confined to the CTCF-bound insulated neighborhood.

Taken together, by focusing on the HS2 enhancer at the human $\beta$-globin gene cluster as a testbed, we provide evidence for the locus-specific epigenetic editing by enCRISPRi through KRAB-mediated H3K9me3 deposition and LSD1-mediated H3K4me1/2 removal. The dual effectors (KRAB and LSD1) act cooperatively to modulate locus-specific epigenetic modifications at the targeted enhancers and associated gene targets.

\section{Allele-Specific Activation of an Oncogenic Super-Enhancer by enCRISPRa}

Having demonstrated the efficacy of the enhanced CRISPR perturbation systems in vitro, we examined whether we could modulate gene transcription and disease phenotypes in vivo by targeting disease-associated CREs. Recurrent mutations at an enhancer 8kb upstream of the TAL1 proto-oncogene were discovered in T-cell acute lymphoblastic leukemia (T-ALL) cell lines and patients ${ }^{44}$. In each case, the heterozygous somatic mutations are acquired through insertion of variable number of nucleotides at the TAL1 enhancer sequences ${ }^{44}$. In Jurkat T-ALL cells, a heterozygous 12bp insertion (GTTAGGAAACGG; Fig. 4a) introduces de novo binding motifs for the MYB proto-oncogene to initiate oncogenic super-enhancer (SE) formation ${ }^{44}$. To establish the proof-of-principle for enCRISPRa in dissecting the in vivo role of TAL1 oncogenic SE in T-ALL, we performed enCRISPRa-mediated activation of TAL1 enhancer in Jurkat cells. We designed two independent sgRNAs that specifically target the mutant allele with protospacer-adjacent motifs (PAM) located within the 12bp insertion sequence (sgMut1 and sgMut2; Fig. 4a). As controls, we designed two sgRNAs targeting the wild-type enhancer sequences in the proximity of the $12 \mathrm{bp}$ insertion sequence (sgWT1 and sgWT2). By stable coexpression of enCRISPRa and individual sgRNAs targeting the TAL1 oncogenic SE in Jurkat cells, we found that TAL1 mRNA and protein were significantly induced by sgRNAs targeting WT or mutant alleles relative to the sgGal4 control (Fig. 4b,c). Since sgMut1 and sgMut2 specifically target the mutant enhancer sequences, these results suggest that allele-specific modulation of TAL1 oncogenic SE allele achieves comparable efficacy on TAL1 transcriptional activation as targeting both alleles.

Given the stronger effects on TAL1 activation by sgMut2 and sgWT2 (Fig. 4a-c), we focused on these sgRNAs for the functional studies of enCRISPRa-mediated activation of TAL1 oncogenic SE in T-ALL cells in vitro and in vivo. Importantly, enhanced TAL1 expression by enCRISPRa led to significantly increased cell growth in vitro by PrestoBlue cell viability assay (see Methods; Fig. 4d), consistent with the oncogenic role of TAL1 in T-ALL cell proliferation ${ }^{44}$. More importantly, upon xenotransplantation into immunodeficient NSG (NOD-scid IL2Rg ${ }^{\text {null }}$ ) mice, enCRISPRa-mediated TAL1 enhancer activation led to greater tumor burden with significantly 
increased bioluminescence signals of the luciferase-expressing T-ALL cells in mice 4 weeks post-transplantation (Fig. 4e,f; see Methods). The mice transplanted with cells expressing TAL1 SE-specific sgRNAs (sgMut2 and sgWT2) also displayed more severe leukemic phenotypes compared to the non-targeting sgGal4, resulting in increased infiltration of T-ALL leukemic cells in peripheral blood (PB) and bone marrow (BM) by flow cytometry and bloodsmear analyses (Fig. 4g,h; see Methods). These results not only establish the functional role of the TAL1 oncogenic SE in promoting T-ALL development, but also demonstrate the efficacy of enCRISPRa for allele-specific activation of disease-associated enhancers in situ and in vivo.

\section{Generation of an Inducible Knock-In Mouse Model for In Vivo enCRISPRi}

Systematic analysis of enhancer function in vivo remains a significant challenge. To explore the in vivo efficacy of enCRISPRi for enhancer perturbation, we engineered a new mouse model by site-specific knock-in (KI) of the dCas9-KRAB chimeric gene under the tetracycline-inducible promoter (TRE) into the Col1a1 locus, which was previously used as a 'safe harbor' for robust transgene expression ${ }^{45,46}$, in $\mathrm{KH} 2$ embryonic stem cells (ESCs) (Fig. 5a; see Methods). $\mathrm{KH} 2$ ESCs also harbor the Rosa26-M2rtTA KI allele allowing expression of the rtTA-M2 transactivator for doxycycline-inducible studies ${ }^{47}$. After blastocyst injection of targeted ESCs and screening of germline transmitted offsprings, the founder mice were crossed to obtain the dCas9-KRAB::Rosa26-M2rtTA heterozygous or homozygous knock-in mice (named dCas9KRAB KI hereafter) (Fig. 5b). The inducible dCas9-KRAB protein expression was confirmed in the targeted $\mathrm{KH} 2 \mathrm{ESC}$ and bone marrow cells isolated from the dCas9-KRAB KI mice (Fig. 5c). To determine the effect of dCas9-KRAB expression on mouse hematopoietic development, we performed complete blood counts of peripheral blood (Fig. S4a) and flow cytometry of various mature hematopoietic cell types including erythroid $\left(\right.$ Ter119 ${ }^{+}$), B-lymphoid $\left(B 220^{+}\right)$, T-lymphoid $\left(\mathrm{CD}^{+}\right)$and myeloid (Mac1+Gr1 ${ }^{+}$) cells in bone marrow and spleen of wild-type (WT) or dCas9KRAB KI mice with or without Dox treatment, respectively (Fig. S4b,c). Our results revealed no overt abnormalities on the frequency of mature hematopoietic cells before or after Dox-induced dCas9-KRAB expression (Fig. S4a-C). Furthermore, the cellularity and frequency of various hematopoietic stem/progenitor cell populations including HSC, MPP, LSK, CMP, GMP and MEP in bone marrow and spleen were comparable in WT and $\mathrm{KI}$ mice with or without Dox treatment (Fig. S5a,b; see Methods), indicating that the inducible dCas9-KRAB expression does not affect normal blood development.

Hematopoiesis serves as a paradigm for understanding stem cell differentiation controlled by lineage-specifying TFs ${ }^{48}$. The self-renewing hematopoietic stem cells (HSCs) give rise to all mature blood cell lineages through a hierarchy of progenitors during lineage specification. In the bone marrow transplantation (BMT) setting, HSCs are capable of reconstituting the entire blood system of a recipient, whereas the short-lived progenitors do not. HSC self-renewal and/or lineage determination are controlled by a small number of TFs, many of which function in a highly lineage-specific manner and are regulated by tissue-specific and/or developmentally 
regulated enhancers ${ }^{48}$. We reasoned that by epigenetic modulation of lineage-specific enhancers in HSCs followed by BMT, we could assay the HSC-derived mature cell lineages as the 'readout' for the functional impact of enhancer perturbations during HSC lineage differentiation in vivo.

\section{In Vivo Functional Interrogation of Lineage-Specific Enhancers}

To this end, we devised an in vivo enhancer perturbation assay by combining the dCas9-KRAB $\mathrm{KI}$ mice with sgRNA-MS2 and MCP-LSD1 to assemble the enCRISPRi complex in vivo (Fig. $5 a)$. We then determined the functional role of lineage-specific enhancers associated with major hematopoietic TFs by in vivo enCRISPRi (Fig. 5d). We focused on five TFs including Cebpa ${ }^{49}$, Spi1 (or PU.1) ${ }^{50}$, Gata1 $^{51}$, Gata2 ${ }^{52,53}$ and Runx1 ${ }^{54,55}$ that play critical roles in HSC function and/or lineage differentiation ${ }^{48}$. Each gene contains one or multiple annotated enhancers based on chromatin accessibility by ATAC-seq and enhancer-associated H3K27ac by ChIP-seq ${ }^{56}$ (Figs. 5e,f, 6c, and S6-S8). We designed 2 or 3 sgRNAs for each enhancer, 2 or 3 sgRNAs for each gene promoter as positive controls, and 10 non-targeting sgRNAs as negative controls (Table S2). The target-specific and control sgRNAs were pooled for each gene for locus-specific enhancer perturbation (total 5 pools with 16 to 20 sgRNAs in each pool). CD45.2 ${ }^{+}$BM hematopoietic stem/progenitor cells (HSPCs) from dCas9-KRAB KI mice were isolated and retrovirally transduced with pooled sgRNAs at $\mathrm{MOI} \leq 0.5$ to ensure that each cell contained no more than one sgRNA (see Methods). The transduced cells were selected and transplanted into CD45. $1^{+}$lethally irradiated recipient mice, followed by Dox administration to induce dCas9KRAB expression for 16 weeks. By this time, all donor-derived hematopoietic cells (CD45.2 $2^{+}$ were differentiated from repopulating donor HSCs instead of short-lived progenitors. We collected cells before BMT (T1) as the baseline control and donor-derived mature myeloid

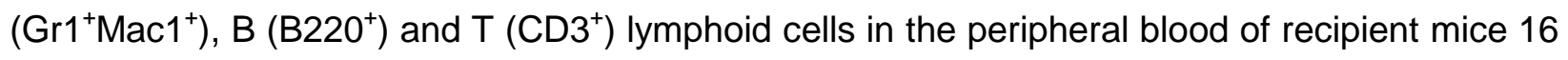
weeks post-BMT (T2). We did not analyze erythroid and megakaryocytic lineages because mature erythroblasts and platelets do not contain nuclei. Genomic DNA from T1 and T2 cells were isolated to amplify the sgRNA sequences, followed by high-throughput amplicon sequencing (see Methods). If enCRISPRi-mediated repression of an enhancer or promoter impaired its function and target gene expression, the corresponding sgRNAs would be depleted (or enriched) in $\mathrm{T} 2$ relative to $\mathrm{T} 1$ cells (Fig. $5 \mathrm{~d}$ ).

We performed five independent locus-specific enhancer perturbation screens, each with two independent replicate experiments, for Cebpa, Spi1, Gata1, Gata2 and Runx1, respectively (Figs. 5e,f, 6c, and S6-S8). Deletion of Cebpa or its downstream enhancer (E4, +37kb from TSS; Fig. 5e) led to defective myeloid-lineage priming and differentiation without affecting lymphopoiesis ${ }^{49,57}$. Consistent with these findings, sgRNAs for E4 enhancer were the top depleted sgRNAs in myeloid cells (ranked \#2 and \#4) next to the positive control sgRNAs for the Cebpa promoter (ranked \#1 and \#3; Fig. 5e) in independent screen experiments (Fig. S6a,b). More importantly, our results revealed that Cebpa E2 (+8kb) enhancers was also required for in 
vivo myelopoiesis, whereas E1 (-20kb) and E3 (+32kb) were dispensable (Figs. 5e and S6a,b). None of the Cebpa enhancers was required for $\mathrm{B}$ or $\mathrm{T}$ cell development considering both the fold changes of sgRNA abundance and the significance of sgRNA depletion between T1 and T2 (Figs. 5e and S6a,b; see Methods). For Spi1 locus, we observed significant depletion of all 3 independent sgRNAs for its $-14 \mathrm{~kb}$ enhancer in myeloid and B but not $\mathrm{T}$ cells (Figs. $5 f$ and S6c,d). These results are consistent with the role of Spi1/PU.1 for normal myeloid and B cell development ${ }^{50,58}$, and validate the in vivo requirement of its upstream enhancer in regulating myelopoiesis and B lymphopoiesis ${ }^{59}$.

With regards to Gata1, while sgRNAs for Gata1 promoters were modestly depleted in myeloid cells consistent with the role of GATA1 in eosinophil and mast cell function ${ }^{48,60}$, none of the sgRNAs for two Gata1 enhancers was depleted in any of the three lineages (Fig. S7a,b). Interestingly, Gata1 enhancers displayed chromatin accessibility and H3K27ac enrichment in HSCs but not mature myeloid, B and T cells, suggesting that the HSC-specific Gata1 enhancers are not required for HSC differentiation to mature myeloid or lymphoid lineages. For Gata2, we observed that an intronic enhancer $(+9.5 \mathrm{~kb}$, E4) was required for in vivo myelopoiesis, whereas the other three gene-distal enhancers were minimally required for any of the three lineages (Fig. S7c,d). It is important to note that the in vivo functional impacts of enhancer perturbation largely correlated with the levels of target gene repression by enCRISPRi-mediated targeting of individual enhancers or promoters in HSPCs (Fig. S9). The lineage-restricted impacts on HSC differentiation also correlated with the presence of enhancer-associated chromatin features (ATAC-seq and H3K27ac) in different lineages (Figs. 5e,f, 6c, and S6-S8), highlighting the context-specific requirements of developmental enhancers in regulating gene expression and lineage differentiation.

\section{Multiplexed Perturbations of Enhancer Function During Hematopoiesis}

Locus-specific perturbation screens provided information for the 'essentiality' of each enhancer within its local context (Figs. 5, S6 and S7), but did not reveal the relative importance of enhancers across multiple loci during hematopoiesis. To address this question, we pooled the target-specific and control sgRNAs for all five hematopoietic TFs, and performed multiplexed enhancer perturbation screens (Fig. 6a). We observed that the top depleted enhancer sgRNAs in myeloid cells included Cebpa $+37 \mathrm{~kb}$ (E4) and $+8 \mathrm{~kb}$ (E2) enhancers, Gata2 $+9.5 \mathrm{~kb}$ (E4) and Spi1 -14kb enhancers when considering the fold changes of sgRNA abundance and the significance of sgRNA depletion (Figs. 6b and S10). The sgRNAs for Spi1 and Gata2 E1, E3 and E4 enhancers were modestly or slightly depleted in B and T cells, respectively (Figs. 6b and S10). These results illustrate the disparate requirements for distinct enhancers during hematopoiesis, with some enhancers broadly required for multiple lineages (e.g. Gata2 or Spi1 enhancers) while others uniquely required for one specific lineage (e.g. Cebpa enhancers). Our results also demonstrate that the in vivo enhancer functions cannot be solely predicted based on enhancer-associated epigenetic features. For example, while all four Cebpa enhancers 
share similar chromatin accessibility and H3K27ac enrichment in HSCs and/or myeloid cells, only $+8 \mathrm{~kb}$ E2 and $+37 \mathrm{~kb}$ E4 enhancers are indispensable for myeloid development (Figs. 5e and $6 b)$.

By locus-specific enhancer perturbation, we found that sgRNAs for Runx1 promoters (P1 and P2) were significantly depleted in B and T cells but enriched in myeloid cells, whereas none of sgRNAs for the annotated Runx1 enhancers showed differential enrichment (Figs. 6c and S8a,b). Similar results were replicated in multi-loci multiplexed perturbation (Figs. $6 \mathrm{~d}$ and $\mathrm{S} 8 \mathrm{c}, \mathrm{d})$. These findings demonstrate that enCRISPRi-mediated repression of Runx1 promoters impaired HSC differentiation to B and T lineages but promoted myeloid differentiation; however, enCRISPRi-mediated enhancer repression was ineffectual, supporting the non-essential roles of Runx1 enhancers in myelopoiesis or lymphopoiesis. More importantly, the opposing phenotypes of repressing Runx1 by enCRISPRi in myeloid vs lymphoid lineages are consistent with the differential roles of Runx1 in hematopoiesis ${ }^{61}$. Hematopoietic cell-specific Runx $1 \mathrm{KO}$ in mice led to the development of myeloproliferative phenotypes characterized by defective $\mathrm{B}$ - and T-cell maturation, and increased myelopoiesis ${ }^{61}$. Our results faithfully recapitulated the phenotypic manifestations of Runx1 deficiency, illustrating the utility of enCRISPRi-based epigenetic editing in functional analysis of cis-regulatory elements during in vivo development. Hence, the exemplified applications of enhancer perturbations in vitro (Figs. 1-3), in xenografts (Fig. 4) and in vivo (Figs. 5,6 ) in multiple cell models establish the enhanced CRISPR epigenetic editing systems as general tools for functional interrogation of non-coding regulatory genome in development and disease.

\section{DISCUSSION}

\section{Targeted Epigenetic Editing In Situ by Dual Effector dCas9 Complexes}

Here we describe the enhanced CRISPR epigenetic editing systems for locus-specific activation or repression of transcriptional enhancers or other CREs in native chromatin. Using epigenetic writer proteins that specifically modulate histone modifications associated with active enhancers, the new systems enable highly efficient and multiplexed analysis of enhancer function in human and mouse cells in vitro, in xenografts and in vivo. Our results also demonstrate that independent repressors (LSD1 and KRAB in enCRISPRi) or activators (p300 and VP64 in enCRISPRa) cooperate to modulate gene transcription by remodeling epigenetic landscapes at the targeted genomic loci. KRAB is associated with heterochromatin formation ${ }^{30}$, whereas LSD1 removes enhancer-associated H3K4me1/2 ${ }^{40}$. The combined effects on H3K9me3 deposition and H3K4me1/2 removal exceeded individual effectors when targeted to the HS2 enhancer (Figs. 3, S1 and S3), suggesting that different epigenetic effectors act cooperatively and/or synergistically for maximal enhancer perturbations. Moreover, the 
development of enCRISPRi and enCRISPRa systems permits parallel analyses of the same loci by both loss- and gain-of-function approaches, thus facilitating the identification of CREs that are necessary and/or sufficient for target gene expression. It is important to note that, while our studies focus on the applications of enCRISPRi and enCRISPRa for modulating enhancer activities, the dual-effector systems also work effectively on modulating gene expression when targeted to gene-proximal promoters in vitro and in vivo (Figs. 1b, 5e,f, 6b-d, S6-S8 and S10).

\section{Considerations for Enhancer Perturbations by CRISPR Epigenetic Editing}

We found that a single sgRNA targeting a single enhancer was sufficient for gene repression; however, the position of sgRNAs can significantly affect the effectiveness of CRISPR epigenetic editing as shown in the case of HS2 enhancer (Fig. 2e,f). Given that we positioned sgRNAs based on the distances to the DHS or ATAC-seq peak summits at enhancers, these results indicate that efficient enhancer perturbations require maximal interference with the chromatin binding of enhancer-regulating TFs. Consistent with this idea, the dual effector enCRISPRi led to more significant disruption of GATA1 and TAL1 binding than single effector dCas9-KRAB or dCas9-LSD1 at HS2 enhancer (Fig. 3). These findings are distinct from CRISPRi in promoter repression, in which targeting sgRNAs to promoter downstream sequences (50 100bp) was most effective in gene repression likely due to the interference with transcription initiation and/or elongation complexes ${ }^{19}$. In future studies, high-resolution tiling screens of critical TF binding site(s) at enhancer sequences may elicit the functional roles of individual TF binding sites in enhancer regulation. Such studies will not only provide insights into the regulatory mechanisms for enhancer function, but also identify selective 'vulnerabilities' of enhancers that may be employed to precisely control gene expression.

\section{In Vivo Interrogation of Enhancer Functions in Development and Disease}

By in vivo multiplexed perturbation screens, we identified several candidate enhancers required for lineage differentiation of HSCs (Figs. 5, 6 and S6-S10). Although the lineage-specific essentiality largely correlated with the presence of enhancer-associated chromatin accessibility and H3K27ac, the functional roles of individual enhancers cannot be reliably predicted based on only epigenetic features. For instances, while the annotated Cebpa enhancers share similar chromatin features, only $+8 \mathrm{~kb}$ E2 and $+37 \mathrm{~kb}$ E4 enhancers are indispensable for Cebpa expression and myeloid development (Figs. 5e, 6b and S6). Similarly, while several annotated Runx1 enhancers harbor enhancer-associated chromatin features in HSCs and/or differentiated lineages, none of them was required for Runx1 expression or function in HSC differentiation to myeloid and lymphoid cells in vivo (Figs. 6 and S8). These results highlight the importance of analyzing enhancer function in situ by loss- and gain-of-function assays, such as the enCRISPRi and enCRISPRa epigenetic editing systems described here, ideally during in vivo development. These assays require the analysis of cellular phenotypes such as stem cell 
differentiation, cell proliferation, viability, and/or response to stimuli as readouts to quantify the functional impact.

Therefore, the development of dCas9-KRAB KI mouse model provides opportunities for in vivo functional interrogation of enhancers and other CREs in lineage differentiation of tissue stem cells. Multiplexed analysis of many genes or loci allows efficient functional screens to prioritize relevant CREs for in-depth characterization. In future studies, the inducible dCas9-KRAB KI mouse model combined with various disease models will provide new insights into the roles of non-coding CREs in disease pathogenesis in vivo. In addition, the improved CRISPR epigenetic editing systems should accelerate functional follow-up studies of disease or trait-associated genetic variants and cancer-associated somatic alterations, many of which reside in non-coding CREs including enhancers. Finally, the enhanced CRISPR epigenetic editing may suggest potential therapeutic strategies by generating targeted epigenetic modifications to alter the expression of desired genes. In conclusion, we have developed dual activator or repressorcontaining CRISPR perturbation systems for functional analysis of non-coding regulatory elements. Our studies not only identify candidate lineage-specific enhancers required for hematopoiesis, but also establish a widely applicable platform for unbiased analysis of noncoding regulatory genome which can be extended to other cell types and human diseases.

\section{AUTHOR CONTRIBUTIONS}

Conceptualization, K.L., Y.L. and J.X.; Methodology, K.L., Y.L., H.C., Y.Z., Z.G., X.L., A.Y., P.K., K.E.D., M.N. and J.X.; Investigation, K.L., X.L., H.C., P.K. and J.X.; Writing - Original Draft, K.L., Y.L. and J.X.; Writing - Review \& Editing, J.X.; Funding Acquisition, J.X.; Supervision, J.X.

\section{ACKNOWLEDGMENTS}

We thank the BioHPC computational infrastructure at UTSW for assistance, Lin Li and Le Qi for technical support, and Luke A. Gilbert, Stanley S. Qi, and Jonathan S. Weissman at UCSF for providing the CRISPRi constructs. K.L. and Y.L. were supported by the Cancer Prevention and Research Institute of Texas (CPRIT) training grant (RP160157). X.L. was supported by the American Heart Association postdoctoral fellowship (18POST34060219). J.X. is a Scholar of The Leukemia \& Lymphoma Society. This work was supported by the NIH grants R01DK111430 and R01CA230631, the CPRIT grants (RR140025, RP180504, RP180826 and RP190417), and the Welch Foundation grant I-1942 (to J.X.). 


\section{REFERENCES}

1. Thurman, R.E. et al. The accessible chromatin landscape of the human genome. Nature 489, 75-82 (2012).

2. Andersson, R. et al. An atlas of active enhancers across human cell types and tissues. Nature 507, 455-461 (2014).

3. Consortium, T.E.P. An integrated encyclopedia of DNA elements in the human genome. Nature 489, 57-74 (2012).

4. Bernstein, B.E. et al. The NIH Roadmap Epigenomics Mapping Consortium. Nature biotechnology 28, 1045-1048 (2010).

5. Heintzman, N.D. et al. Distinct and predictive chromatin signatures of transcriptional promoters and enhancers in the human genome. Nature genetics 39, 311-318 (2007).

6. Visel, A., Rubin, E.M. \& Pennacchio, L.A. Genomic views of distant-acting enhancers. Nature 461, 199-205 (2009).

7. Ong, C.T. \& Corces, V.G. Enhancer function: new insights into the regulation of tissue-specific gene expression. Nature reviews. Genetics 12, 283-293 (2011).

8. Banerji, J., Rusconi, S. \& Schaffner, W. Expression of a beta-globin gene is enhanced by remote SV40 DNA sequences. Cell 27, 299-308 (1981).

9. Arnold, C.D. et al. Genome-wide quantitative enhancer activity maps identified by STARR-seq. Science (New York, N.Y.) 339, 1074-1077 (2013).

10. Vockley, C.M. et al. Massively parallel quantification of the regulatory effects of noncoding genetic variation in a human cohort. Genome research 25, 1206-1214 (2015).

11. Nord, A.S. et al. Rapid and pervasive changes in genome-wide enhancer usage during mammalian development. Cell 155, 1521-1531 (2013).

12. Bauer, D.E. et al. An erythroid enhancer of BCL11A subject to genetic variation determines fetal hemoglobin level. Science (New York, N.Y.) 342, 253-257 (2013).

13. Huang, J. et al. Dynamic Control of Enhancer Repertoires Drives Lineage and Stage-Specific Transcription during Hematopoiesis. Developmental Cell 36, 9-23 (2016).

14. Canver, M.C. et al. BCL11A enhancer dissection by Cas9-mediated in situ saturating mutagenesis. Nature 527, 192-197 (2015).

15. Korkmaz, G. et al. Functional genetic screens for enhancer elements in the human genome using CRISPR-Cas9. Nature biotechnology 34, 192-198 (2016).

16. Sanjana, N.E. et al. High-resolution interrogation of functional elements in the noncoding genome. Science (New York, N.Y.) 353, 1545-1549 (2016).

17. Qi, L.S. et al. Repurposing CRISPR as an RNA-guided platform for sequence-specific control of gene expression. Cell 152, 1173-1183 (2013).

18. Gilbert, L.A. et al. CRISPR-mediated modular RNA-guided regulation of transcription in eukaryotes. Cell 154, 442-451 (2013).

19. Gilbert, L.A. et al. Genome-Scale CRISPR-Mediated Control of Gene Repression and Activation. Cell 159, 647-661 (2014).

20. Zalatan, J.G. et al. Engineering complex synthetic transcriptional programs with CRISPR RNA scaffolds. Cell 160, 339-350 (2015).

21. Konermann, S. et al. Genome-scale transcriptional activation by an engineered CRISPR-Cas9 complex. Nature 517, 583-588 (2015).

22. Hilton, I.B. et al. Epigenome editing by a CRISPR-Cas9-based acetyltransferase activates genes from promoters and enhancers. Nature biotechnology 33, 510-517 (2015).

23. Thakore, P.I. et al. Highly specific epigenome editing by CRISPR-Cas9 repressors for silencing of distal regulatory elements. Nature methods 12, 1143-1149 (2015).

24. Hu, J.H. et al. Evolved Cas9 variants with broad PAM compatibility and high DNA specificity. Nature 556, 57-63 (2018).

25. Liu, X.S. et al. Editing DNA Methylation in the Mammalian Genome. Cell 167, 233-247.e217 (2016).

26. Amabile, A. et al. Inheritable Silencing of Endogenous Genes by Hit-and-Run Targeted Epigenetic Editing. Cell 167, 219-232.e214 (2016).

27. Yeo, N.C. et al. An enhanced CRISPR repressor for targeted mammalian gene regulation. Nature methods 15, 611-616 (2018). 
28. Klann, T.S. et al. CRISPR-Cas9 epigenome editing enables high-throughput screening for functional regulatory elements in the human genome. Nature biotechnology 35, 561-568 (2017).

29. Kearns, N.A. et al. Functional annotation of native enhancers with a Cas9-histone demethylase fusion. Nature methods 12, 401-403 (2015).

30. Urrutia, R. KRAB-containing zinc-finger repressor proteins. Genome biology 4, 231 (2003).

31. Peabody, D.S. The RNA binding site of bacteriophage MS2 coat protein. The EMBO journal 12, 595600 (1993).

32. Visel, A. et al. ChIP-seq accurately predicts tissue-specific activity of enhancers. Nature 457, $854-$ 858 (2009).

33. Heintzman, N.D. et al. Histone modifications at human enhancers reflect global cell-type-specific gene expression. Nature 459, 108-112 (2009).

34. Creyghton, M.P. et al. Histone H3K27ac separates active from poised enhancers and predicts developmental state. Proceedings of the National Academy of Sciences of the United States of America 107, 21931-21936 (2010).

35. Liu, X. et al. In Situ Capture of Chromatin Interactions by Biotinylated dCas9. Cell 170, 10281043.e1019 (2017).

36. Li, Q., Peterson, K.R., Fang, X. \& Stamatoyannopoulos, G. Locus control regions. Blood 100, 30773086 (2002).

37. Dominguez, A.A., Lim, W.A. \& Qi, L.S. Beyond editing: repurposing CRISPR-Cas9 for precision genome regulation and interrogation. Nature reviews. Molecular cell biology 17, 5-15 (2016).

38. Thakore, P.I., Black, J.B., Hilton, I.B. \& Gersbach, C.A. Editing the epigenome: technologies for programmable transcription and epigenetic modulation. Nature methods 13, 127-137 (2016).

39. Chavez, A. et al. Comparison of Cas9 activators in multiple species. Nature methods 13, 563-567 (2016).

40. Shi, Y. et al. Histone demethylation mediated by the nuclear amine oxidase homolog LSD1. Cell 119, 941-953 (2004).

41. Williams, R.M. et al. Genome and epigenome engineering CRISPR toolkit for in vivo modulation of cis-regulatory interactions and gene expression in the chicken embryo. Development (Cambridge, England) 145 (2018).

42. Deng, W. et al. Reactivation of developmentally silenced globin genes by forced chromatin looping. Cell 158, 849-860 (2014).

43. Sauvageau, M. \& Sauvageau, G. Polycomb group proteins: multi-faceted regulators of somatic stem cells and cancer. Cell stem cell 7, 299-313 (2010).

44. Mansour, M.R. et al. Oncogene regulation. An oncogenic super-enhancer formed through somatic mutation of a noncoding intergenic element. Science (New York, N.Y.) 346, 1373-1377 (2014).

45. Carey, B.W., Markoulaki, S., Beard, C., Hanna, J. \& Jaenisch, R. Single-gene transgenic mouse strains for reprogramming adult somatic cells. Nature methods 7, 56-59 (2010).

46. Stadtfeld, M., Maherali, N., Borkent, M. \& Hochedlinger, K. A reprogrammable mouse strain from gene-targeted embryonic stem cells. Nature methods 7, 53-55 (2010).

47. Hochedlinger, K., Yamada, Y., Beard, C. \& Jaenisch, R. Ectopic expression of Oct-4 blocks progenitor-cell differentiation and causes dysplasia in epithelial tissues. Cell 121, 465-477 (2005).

48. Orkin, S.H. \& Zon, L.I. Hematopoiesis: an evolving paradigm for stem cell biology. Cell 132, 631-644 (2008).

49. Zhang, D.E. et al. Absence of granulocyte colony-stimulating factor signaling and neutrophil development in CCAAT enhancer binding protein alpha-deficient mice. Proceedings of the National Academy of Sciences of the United States of America 94, 569-574 (1997).

50. Klemsz, M.J., McKercher, S.R., Celada, A., Van Beveren, C. \& Maki, R.A. The macrophage and B cell-specific transcription factor PU.1 is related to the ets oncogene. Cell 61, 113-124 (1990).

51. Tsai, S.F. et al. Cloning of cDNA for the major DNA-binding protein of the erythroid lineage through expression in mammalian cells. Nature 339, 446-451 (1989).

52. Zon, L.I. et al. Expression of GATA-binding proteins during embryonic development in Xenopus laevis. Proceedings of the National Academy of Sciences of the United States of America 88, 1064210646 (1991).

53. Lee, M.E., Temizer, D.H., Clifford, J.A. \& Quertermous, T. Cloning of the GATA-binding protein that regulates endothelin-1 gene expression in endothelial cells. The Journal of biological chemistry $\mathbf{2 6 6}$, 16188-16192 (1991). 
54. Wang, S.W. \& Speck, N.A. Purification of core-binding factor, a protein that binds the conserved core site in murine leukemia virus enhancers. Molecular and cellular biology 12, 89-102 (1992).

55. Okuda, T., van Deursen, J., Hiebert, S.W., Grosveld, G. \& Downing, J.R. AML1, the target of multiple chromosomal translocations in human leukemia, is essential for normal fetal liver hematopoiesis. Cell 84, 321-330 (1996).

56. Lara-Astiaso, D. et al. Immunogenetics. Chromatin state dynamics during blood formation. Science (New York, N.Y.) 345, 943-949 (2014).

57. Avellino, R. et al. An autonomous CEBPA enhancer specific for myeloid-lineage priming and neutrophilic differentiation. Blood 127, 2991-3003 (2016).

58. Iwasaki, H. et al. Distinctive and indispensable roles of PU.1 in maintenance of hematopoietic stem cells and their differentiation. Blood 106, 1590-1600 (2005).

59. Rosenbauer, F. et al. Acute myeloid leukemia induced by graded reduction of a lineage-specific transcription factor, PU.1. Nature genetics 36, 624-630 (2004).

60. Yu, C. et al. Targeted deletion of a high-affinity GATA-binding site in the GATA-1 promoter leads to selective loss of the eosinophil lineage in vivo. The Journal of experimental medicine 195, 13871395 (2002).

61. Growney, J.D. et al. Loss of Runx1 perturbs adult hematopoiesis and is associated with a myeloproliferative phenotype. Blood 106, 494-504 (2005).

62. Beard, C., Hochedlinger, K., Plath, K., Wutz, A. \& Jaenisch, R. Efficient method to generate singlecopy transgenic mice by site-specific integration in embryonic stem cells. Genesis (New York, N.Y. : 2000) 44, 23-28 (2006).

63. Gu, Z. et al. Loss of EZH2 Reprograms BCAA Metabolism to Drive Leukemic Transformation. Cancer discovery (2019).

64. $\mathrm{Xu}, \mathrm{J}$. et al. Combinatorial assembly of developmental stage-specific enhancers controls gene expression programs during human erythropoiesis. Developmental Cell 23, 796-811 (2012).

65. Xu, J. et al. Correction of sickle cell disease in adult mice by interference with fetal hemoglobin silencing. Science (New York, N.Y.) 334, 993-996 (2011).

66. Dobin, A. et al. STAR: ultrafast universal RNA-seq aligner. Bioinformatics (Oxford, England) 29, 1521 (2013).

67. Li, B. \& Dewey, C.N. RSEM: accurate transcript quantification from RNA-Seq data with or without a reference genome. BMC bioinformatics 12, 323 (2011).

68. Love, M.I., Huber, W. \& Anders, S. Moderated estimation of fold change and dispersion for RNA-seq data with DESeq2. Genome biology 15, 550 (2014).

69. Langmead, B., Trapnell, C., Pop, M. \& Salzberg, S.L. Ultrafast and memory-efficient alignment of short DNA sequences to the human genome. Genome biology 10, R25 (2009).

70. Zhang, Y. et al. Model-based analysis of ChIP-Seq (MACS). Genome biology 9, R137 (2008).

71. Shao, Z., Zhang, Y., Yuan, G.C., Orkin, S.H. \& Waxman, D.J. MAnorm: a robust model for quantitative comparison of ChIP-Seq data sets. Genome biology 13, R16 (2012).

72. Li, W. et al. MAGeCK enables robust identification of essential genes from genome-scale CRISPR/Cas9 knockout screens. Genome biology 15, 554 (2014). 


\section{METHODS}

\section{Cells and Cell Culture}

Human K562 and Jurkat cells were obtained from ATCC and cultured RPMI1640 medium containing 10\% fetal bovine serum (FBS), 1\% penicillin/streptomycin (P/S). Human HEK293T cells were obtained from ATCC and cultured in DMEM medium containing 10\% FBS and $1 \%$ $\mathrm{P} / \mathrm{S}$. KH2 ESCs were cultured in DMEM medium containing 10\% ES-certified FBS (GemCell ${ }^{\mathrm{TM}}$, Cat\# 100-500), 1\% P/S, 2mM L-Glutamine (Gibco), 0.1mM MEM non-essential amino acids solution (Gibco), 1mM sodium pyruvate (Gibco), 0.1mM $\beta$-Mercaptoethanol and 1000U/ml recombinant mouse LIF (ESGRO, Cat\# ESG1107). All cultures were incubated at $37^{\circ} \mathrm{C}$ in $5 \%$ $\mathrm{CO}_{2}$. No cell line used in this study was found in the database of commonly misidentified cell lines that is maintained by ICLAC and NCBI BioSample. All cell lines were tested for mycoplasma contamination.

\section{Plasmids}

To generate the inducible dCas9-p300 expression vector, the p300 HAT core domain (p300 core) was PCR amplified from the pcDNA-dCas9-p300-Core vector (Addgene, Plasmid \#61357) and cloned into Mlul/BstXI digested pHR-TRE3G-KRAB-dCas9-P2A-mCherry backbone, which was a gift from Luke A. Gilbert ${ }^{23}$. To generate the inducible dCas9-LSD1 expression vector, LSD1 open-reading frame (ORF) was amplified and cloned into the pHR-TRE3G-KRAB-dCas9P2A-mCherry to replace the KRAB domain. To generate the enCRISPRa sgRNA vector, the MCP-VP64-IRES-mCherry cassette was PCR amplified from the pJZC34 vector (Addgene, plasmid \# 62331) and cloned into BsrGI/EcoRI digested lenti-sgRNA (MS2)-zeo backbone (Addgene, plasmid \# 61427). Then the mCherry cassette was replaced by Puro or EGFP by InFusion ${ }^{\circledR}$ HD Cloning Kit (Clontech). To generate the enCRISPRi-LK sgRNA vector, the KRAB sequence was PCR amplified from the pLV hU6-sgRNA hUbC-dCas9-KRAB-T2A-Puro vector (Addgene, plasmid \#71236) and cloned into the enCRISPRa sgRNA vector to replace VP64. To generate enCRISPRi-KL sgRNA vector, MCP-LSD1 was amplified and cloned into pMLSNRAS-T2A-GFP-polyA-U6 to replace NRAS.

\section{Design and Cloning of sgRNAs}

sgRNAs were designed to minimize off-targets based on publicly available filtering tools (http://crispr.genome-engineering.org/crispr/). Briefly, oligonucleotides were annealed in the following reaction: $10 \mu \mathrm{M}$ guide sequence oligo, 10 $\mu \mathrm{M}$ reverse complement oligo, T4 ligation buffer (1X), and $5 \mathrm{U}$ of T4 polynucleotide kinase (NEB) with the cycling parameters of $37^{\circ} \mathrm{C}$ for $30 \mathrm{~min} ; 95^{\circ} \mathrm{C}$ for $5 \mathrm{~min}$ and then ramp down to $25^{\circ} \mathrm{C}$ at $5^{\circ} \mathrm{C} / \mathrm{min}$. The annealed oligos were cloned into the sgRNA vectors using a Golden Gate Assembly strategy including: 100ng of circular sgRNA vector plasmid, $0.2 \mu \mathrm{M}$ annealed oligos, buffer 2.1 (1X) (NEB), $20 \mathrm{U}$ of Bbsl restriction enzyme, $0.2 \mathrm{mM}$ ATP, $0.1 \mathrm{mg} / \mathrm{ml} \mathrm{BSA}$, and $750 \mathrm{U}$ of T4 DNA ligase (NEB) with the cycling parameters of 20 cycles at $37^{\circ} \mathrm{C}$ for $5 \mathrm{~min}, 20^{\circ} \mathrm{C}$ for $5 \mathrm{~min}$; followed by $80^{\circ} \mathrm{C}$ incubation for 20min. Insertion of sgRNA was validated by Sanger sequencing. Lentiviruses containing 
sgRNAs were packaged in HEK293T cells. Briefly, $2 \mu \mathrm{g}$ of $\mathrm{p} \Delta 8.9,1 \mu \mathrm{g}$ of VSV-G and $5 \mu \mathrm{g}$ sgRNA vectors were co-transfected into HEK293T cells seeded in $10 \mathrm{~cm}$ petri dish. Lentiviruses were harvested from the supernatant 48 72 $\mathrm{h}$ post-transfection. Dox-Inducible enCRISPRi or enCRISPRa cell lines were then transduced with sgRNA-expressing lentiviruses in 6-well plates. To maximize sgRNA expression, top 1-5\% of GFP-positive cells were FACS sorted $48 \mathrm{~h}$ post-infection. The sequences for all sgRNAs are listed in Table S2.

\section{Generation of Tetracycline-Inducible dCas9-KRAB Knock-In Mouse Model}

Site-specific knock-in (KI) of tetracycline-inducible dCas9-KRAB transgene was generated by flippase (FLPe)-mediated recombination as previously described ${ }^{35,62}$. $\mathrm{KH} 2$ mouse embryonic stem cells (ESCs) harboring the M2rtTA tetracycline-responsive trans-activator in Rosa26 locus and an engineered Col1a1 locus with an frt site and ATG-less hygromycin resistance gene were used ${ }^{62}$. A targeting construct pBS3.1-TRE-dCas9-KRAB containing the PGK promoter, an frt site, a tetracycline-inducible minimal CMV promoter, the dCas9-KRAB transgene, and an ATG initiation codon was co-electroporated with the pCAGGS-FLPe-puro into $\mathrm{KH} 2$ ESCs at 500V and $25 \mu \mathrm{F}$ using a Gene Pulser II (Bio-Rad). Cells were selected with hygromycin $(140 \mu \mathrm{g} / \mathrm{ml})$, and positive clones were expanded and analyzed by genotyping PCR. Targeted ESC clones were injected to embryonic day 3.5 mouse blastocysts to obtain the founder mice. Chimeric founder mice were bred with C57BL/6 mice, and offsprings with germline transmission were genotyped using primers in Table S2 and intercrossed to generate dCas9-KRAB heterozygous or homozygous $\mathrm{KI}$ mice. All mouse experiments were performed under protocols approved by the Institutional Animal Care and Use Committee of UT Southwestern Medical Center (UTSW).

\section{Xenograft Experiments}

Luciferase cassette was amplified and cloned into pLVX-Puro vector (Clontech, Catalog No. 632164). Lentivirus was produced to transduce Jurkat cells co-expressing enCRISPRa with control sgGal4, sgWT2 or sgMut2, respectively. Puromycin selection $(1 \mu \mathrm{g} / \mathrm{ml})$ was performed 3 days after infection. Six to eight weeks old female NOD-SCID (NSG) mice were sub-lethally irradiated $(2.5 \mathrm{~Gy})$ half day before the transplantation. Cells $\left(1 \times 10^{6} / \mathrm{mice}\right)$ were resuspended in PBS $(200 \mu \mathrm{l} / \mathrm{mice})$ and intravenously transplanted. Transplanted mice underwent in vivo bioluminescence imaging at various time points to evaluate tumor growth. Briefly, following intraperitoneal injection of $150 \mathrm{mg} / \mathrm{kg}$ D-luciferin (Gold Biotechnology), mice were imaged, and bioluminescence intensity was quantitated using Living Image 3.2 acquisition and analysis software (Caliper Life Sciences). Total flux values were determined by drawing regions of interest (ROI) of identical size over each mouse and were presented in photons $(p)$ /second (sec). Four weeks after transplantation, the peripheral blood, bone marrow and spleen were assessed for engraftment by flow cytometry. Bloodsmear was performed and stained with MayGrunwald-Giemsa as previously described ${ }^{63}$. The blue stained cells indicated the circulating leukemia cells.

\section{Generation of Dox-Inducible enCRISPRi or enCRISPRa Cell Lines}


To generate inducible enCRISPRi and enCRISPRa stable cell lines, the target cells were transduced with lentivirus expressing dCas9-KRAB, dCas9-LSD1 or dCas9-p300 and rtTA. Doxycycline was added following infection and flow cytometry was used to sort cells that expressed mCherry and BFP. These cells were then grown in the absence of doxycycline until mCherry fluorescence returned to uninduced levels. Transient transfections were performed in 24-well plates using 500ng of dCas9 expression vector and 250ng of equimolar pooled or individual sgRNA expression vectors mixed with FuGENE $^{\circledR} 6$ (Promega) following manufacturer's instructions.

\section{Cell Growth Assays}

Cell proliferation was determined using the PrestoBlue Cell Viability Reagent (Invitrogen). 5,000 cells/well were seeded in triplicate into 96-well plates. After various days of culture, $10 \mu$ of PrestoBlue reagents were added to wells with cells or medium (blank), relative absorption values at 570 and $600 \mathrm{~nm}$ were read after 1 hour incubation at $37^{\circ} \mathrm{C}$.

\section{RNA Isolation and qRT-PCR Analysis}

RNA was isolated using RNeasy Plus Mini Kit (Qiagen) following manufacturer's protocols. For qRT-PCR, RNA was reverse-transcribed using iScript cDNA Synthesis Kit (Bio-Rad) following manufacturer's protocols. Quantitative RT-PCR (qRT-PCR) was performed in duplicate with the iQ SYBR Green Supermix (Bio-Rad) using CFX384 Touch Real-Time PCR Detection System (Bio-Rad). PCR amplification parameters were $95^{\circ} \mathrm{C}(3 \mathrm{~min})$ and 45 cycles of $95^{\circ} \mathrm{C}(15 \mathrm{sec}$ ), $60^{\circ} \mathrm{C}(30 \mathrm{sec})$, and $72^{\circ} \mathrm{C}$ (30 sec). Primer sequences are listed in Table S2.

\section{Western Blot Analysis}

Western blot was performed as described ${ }^{64}$ using the following antibodies: TAL1 (Santa Cruz Biotechnology, sc-12984), $\beta$-tubulin (Cell Signaling, 2128), HA (Santa Cruz Biotechnology, sc805), and Cas9 (Abcam, ab191468). All antibodies were used at 1:1,000 dilutions. Densitometry quantification was performed using ImageJ software.

\section{Phenotypic Analysis of Hematopoiesis}

Blood was collected via the retro-orbital plexus and complete blood counts (CBC) were performed on a HEMAVET HV950 (Drew Scientific) according to the manufacturer's protocol. Cytospin preparations were stained with May-Grunwald-Giemsa as described previously ${ }^{65}$. BM cells were obtained by crushing femurs, tibias, vertebrae and pelvic bones with a mortar in $\mathrm{Ca}^{2+}$ and $\mathrm{Mg}^{2+}$-free Hank's buffered salt solution (HBSS, Gibco) supplemented with $2 \%$ heatinactivated bovine serum (HIBS, Gibco). Spleens were dissociated by crushing followed by trituration. All BM and spleen cell suspensions were filtered through $70 \mu \mathrm{m}$ cell strainers, followed by cell counting using a Vi-CELL cell viability analyser (Beckman Coulter). For flow cytometric analysis, cells were incubated with combinations of fluorophore-conjugated antibodies. Lineage markers for HSCs and progenitors were CD2, CD3, CD5, CD8, B220, Gr1 and Ter119. Antibody staining was performed at $4{ }^{\circ} \mathrm{C}$ for $30 \mathrm{~min}$ or on ice for $90 \mathrm{~min}$. 
Biotinylated antibodies were visualized by incubation with PE/Cy7-conjugated streptavidin at $4^{\circ} \mathrm{C}$ for $30 \mathrm{~min}$. DAPI (4,6-diamidino-2-phenylindole; $2 \mu \mathrm{g} / \mathrm{ml}$ in PBS) was used to exclude dead cells. The hematopoietic stem/progenitor cell populations in mouse bone marrow were analyzed as previously described ${ }^{63}$, including HSC ( Lin $\left.^{-} \mathrm{Scal}^{+} \mathrm{Kit}^{+} \mathrm{CD} 150^{+} \mathrm{CD} 48^{-}\right)$, MPP (Lin ${ }^{-}$

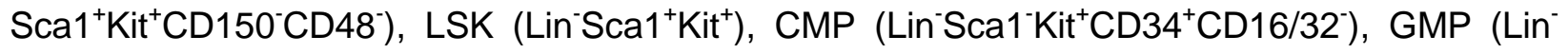

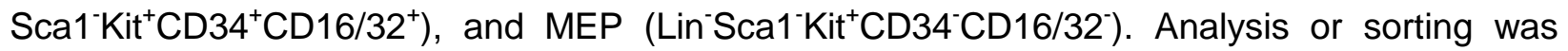
performed using a FACSAria or FACSCanto flow cytometer (BD Biosciences). Data were analyzed using FACSDiva (BD Biosciences).

The following antibodies were used for flow cytometry: PerCP/Cy5.5 anti-mouse B220 (Biolegend, Cat\# 103236), PE anti-mouse CD150 (Biolegend, Cat\# 115903), BV510 anti-mouse CD16/32 (Biolegend, Cat\# 101333), Alexa Fluor 700 anti-mouse CD3 (Biolegend, Cat\# 100216), Biotin anti-mouse CD34 (eBioscience, Cat\# 13-0341-85), PE anti-mouse CD43 (eBioscience, Cat\# 12-0431-83), Alexa Fluor 700 anti-mouse CD48 (Biolegend, Cat\# 103426), FITC anti-mouse CD71 (BD Biosciences, Cat\# 553266), APC anti-mouse c-Kit (Biolegend, Cat\# 105811), PE/Cy7 anti-mouse Gr1 (Biolegend, Cat\# 108415), APC anti-mouse IgM (Biolegend, Cat\# 406509), APC-eFluor 780 anti-mouse Mac1 (eBioscience, Cat\# 47-0112-82), PerCP/Cy5.5 anti-mouse Sca-1 (Biolegend, Cat\# 108123), BV510 anti-mouse Ter119 (Biolegend, Cat\# 116237), FITC anti-mouse CD2 (eBioscience, 11-0021-81), FITC anti-mouse CD3 (Biolegend, Cat\# 100204), FITC anti-mouse CD5 (Biolegend, Cat\# 100606), FITC anti-mouse CD8a (Biolegend, Cat\# 100706), FITC anti-mouse B220 (eBioscience, 11-0452-85), FITC anti-mouse Gr-1 (Biolegend, Cat\# 108406), FITC anti-mouse Ter119 (Biolegend, Cat\# 116206), PE/Cy7 Streptavidin (Biolegend, Cat\# 405206).

\section{RNA-seq and Data Analysis}

RNA-seq library was prepared using the Ovation RNA-seq system (NuGEN). Sequencing reads from all RNA-seq experiments were aligned to human (hg19) reference genome by STAR 2.5.2b ${ }^{66}$ with the parameters: --outFilterMultimapNmax 1. RSEM was used to calculate normalized gene expression (Transcripts per Million Reads or TPM) ${ }^{67}$. Differential gene expression analysis was performed by DESeq ${ }^{68}$.

\section{ChIP-seq and Data Analysis}

ChIP-seq was performed as described ${ }^{35}$ using antibodies for HA (Santa Cruz Biotechnology, sc-805), Cas9 (Abcam, ab191468), H3K4me1 (Abcam, ab8895), H3K4me2 (Millipore, 07-030), H3K27ac (Abcam, ab4729), H3K9me3 (Abcam, ab8898), GATA1 (Abcam, ab11852), TAL1 (Santa Cruz Biotechnology, sc-12984), and CTCF (Millipore, 07-729) in HEK293T and/or K562 cells, respectively. Briefly, cross-linked chromatin was sonicated in RIPA 0 buffer (10mM Tris$\mathrm{HCl}, 1 \mathrm{mM}$ EDTA, $0.1 \%$ sodium deoxycholate, $0.1 \%$ SDS, $1 \%$ Triton $\mathrm{X}-100,0.25 \%$ Sarkosyl, pH8.0) to 200 500bp. Final concentration $150 \mathrm{mM} \mathrm{NaCl}$ was added to the chromatin and antibody mixture before incubation overnight at $4^{\circ} \mathrm{C}$. Chromatin was washed and ChIP DNA was purified. ChIP-seq libraries were generated using NEBNext Ultra II DNA library prep kit following 
the manufacturer's protocol (NEB), and sequenced on an Illumina NextSeq500 system using the 75bp high output sequencing kit. ChIP-seq raw reads were aligned to the human hg19 genome assembly using Bowtie2 ${ }^{69}$ with the default parameters. Only tags that uniquely mapped to the genome were used for further analysis. ChIP-seq peaks were called by MACS using the "--nomodel" parameter ${ }^{70}$. Peaks that overlap with the ENCODE blacklist regions ${ }^{3}$ or the validated non-targeting sgRNA (sgGal4) enriched regions (chr6:74,229,700-74,231,800; chr3:17,443,100-17,444,200 and chr15:68,131,900-68,133,000) were removed. To compare ChIP-seq signal intensities in samples prepared from cells expressing the target-specific sgRNAs versus the non-targeting sgGal4, MAnorm ${ }^{71}$ was applied to remove systematic bias between samples and then calculate the normalized ChIP-seq read densities of each peak for all samples. The window size was $1000 \mathrm{bp}$ which matched the average width of the identified ChIP-seq peaks.

\section{In Vivo Enhancer Perturbation Screen and Data Analysis}

CD45.2 $2^{+} \mathrm{BM}$ lineage negative HSPCs from dCas9-KRAB KI mice were isolated by LS columns (Miltenyi Biotec) and cultured in S-clone SF-O3 medium (Iwai North America) containing 1\%

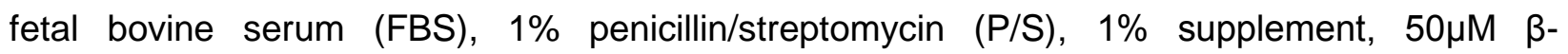
Mercaptoethanol, 50ng/ml mouse SCF and 50ng/ml mouse TPO. After overnight culture, cells were transduced with retroviruses containing target-specific or non-targeting control sgRNAs at $\mathrm{MOI} \leq 0.5$. Twenty million cells per mouse were transplanted into CD45.1 $1^{+}$lethally irradiated C57BL/6 recipient mice, followed by Dox $(2 \mathrm{mg} / \mathrm{ml}$, supplemented with sucrose at $10 \mathrm{mg} / \mathrm{ml})$ administration in drinking water to induce dCas9-KRAB expression for 16 weeks. By this time, all donor-derived hematopoietic cells $\left(C D 45.2^{+}\right)$were differentiated from transplanted donor HSCs instead of short-lived progenitors. We collected cells before bone marrow transplantation (T1) as the baseline control and the GFP positive donor-derived mature myeloid $\left(\mathrm{Gr}^{+} \mathrm{Mac1}{ }^{+}\right), \mathrm{B}$

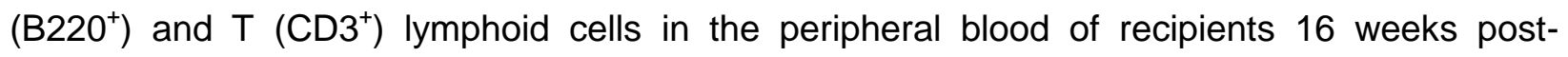
transplantation (T2). Genomic DNA was isolated and sgRNA sequences were PCR amplified using primers listed in Table S2. PCR amplicon libraries were generated using NEBNext Ultra II DNA library prep kit following the manufacturer's protocol (NEB), and sequenced on an Illumina NextSeq500 system using the 75bp high output sequencing kit. Two biological replicate experiments for each enCRISPRi screen were performed. In each replicate experiment, myeloid, B and T cells were isolated from 3 (for locus-specific perturbation) or 15 (for multi-loci perturbation) independent recipient mice, and genomic DNA were isolated and pooled for the sgRNA amplicon sequencing analysis. After sequencing, the sgRNA sequences were extracted from raw fastq files and mapped to sgRNA sequences in enCRISPRi screen libraries. Reads of each sgRNA were counted and normalized to the total read counts of each sample. Mean sgRNA counts from replicates were calculated at starting (T1) and end time point (T2). The growth phenotype of each sgRNA was quantified as log2 transformed mean sgRNA count ratio between T2 and T1. For calculation of the significance ( $P$ value) of depletion for each targeted 
enhancer, promoter or non-targeting control (NC) regions in each cell type, we used MAGECK

72 test with the parameters of --norm-method total --gene-Ifc-method mean.

\section{QUANTIFICATION AND STATISTICAL ANALYSIS}

Statistical details including $N$, mean and statistical significance values are indicated in the text, figure legends, or methods. Error bars in the experiments represent standard error of the mean (SEM) or standard deviation (SD) from either independent experiments or independent samples. All statistical analyses were performed using GraphPad Prism, and the detailed information about statistical methods is specified in figure legends or methods.

\section{REPORTING SUMMARY}

Further information on experimental design is available in the Nature Research Reporting Summary linked to this article.

\section{DATA AND SOFTWARE AVAILABILITY}

All raw and processed ATAC-seq, ChIP-seq and RNA-seq data are available in the Gene Expression Omnibus (GEO): GSE132216. Computation codes are available from the corresponding author on request.

\section{COMPETING FINANCIAL INTERESTS}

The authors declare no competing financial interests. 


\section{FIGURE LEGENDS}

\section{Figure 1. Development of the Dual-Activator enCRISPRa system}

(a) Schematic of the enCRISPRa system containing three components: a dCas9-p300 fusion protein, the target-specific sgRNA with two MS2 hairpins, and the MCP-VP64 fusion protein.

(b) Expression of MYOD, ILIRN and OCT4 upon dxCas9-VPR, SunTag, SAM or enCRISPRamediated enhancer or promoter activation in HEK293T cells. mRNA expression relative to non-transduced cells is shown as mean \pm SEM. The differences between control and dCas 9 activators were analyzed by a two-way ANOVA. ${ }^{\#} P<0.05,{ }^{\# \#} P<0.01,{ }^{\# \# \#} P<0.001$. The difference between enCRISPRa and other dCas9 activators were analyzed by a two-way ANOVA. ${ }^{*} P<0.05,{ }^{\star \star} P<0.01,{ }^{\star \star \star} P<0.001$, n.s. not significant.

(c) Expression of $\beta$-globin genes HBE1, HBG1/2 and HBB upon dxCas9-VPR, SunTag, SAM or enCRISPRa-mediated activation of the HS2 enhancer in HEK293T cells. mRNA expression relative to non-transduced cells is shown as mean \pm SEM and analyzed by a two-way ANOVA.

(d) Genome-wide analysis of dCas9 binding in HEK293T cells expressing HS2-specific sgRNA (two replicate ChIP-seq experiments sgHS2-rep1 and sgHS2-rep2) or non-targeting sgGal4. Data points for the sgRNA target regions and the predicted off-targets are shown as green and red, respectively. The $\mathrm{x}$ - and $\mathrm{y}$-axis denote the normalized read counts (left) or mean normalized read counts from $N=2$ independent ChIP-seq experiments (right).

\section{Figure 2. Development of the Dual-Repressor enCRISPRi system}

(a) Schematic of the enCRISPRi system containing a dCas9-LSD1 fusion protein, the targetspecific sgRNA with two MS2 hairpins, and the MCP-KRAB fusion protein.

(b) Expression of $\beta$-globin genes in K562 cells upon dCas9-KRAB (K), dCas9-LSD1 (L) or enCRISPRi ( $L K$ and $K L$ )-mediated repression of the HS2 enhancer using four independent HS2-targeting sgRNAs individually (sgHS2-1 to sgHS2-4) or combined (sgHS2-all). mRNA expression relative to non-transduced $\mathrm{K} 562$ cells is shown as mean \pm SEM and analyzed by a two-way ANOVA. ${ }^{\star} P<0.05,{ }^{\star \star} P<0.01,{ }^{* \star *} P<0.001$, n.s. not significant.

(c) RNA-seq profiles in K562 cells upon dCas9-KRAB, dCas9-LSD1 or enCRISPRi-mediated repression of the HS2 enhancer using all four HS2-targeting sgRNAs (sgHS2-all). Scatter plot is shown for each gene by the mean of log2 normalized RNA-seq signals as transcripts per million or TPM ( $N=2$ independent experiments) (x-axis) and log2 fold changes of mean TPM in cells expressing sgHS2 and control sgGal4 (y-axis). The $\beta$-globin genes (HBE1, $H B G 1, H B G 2$ and $H B B$ ) are indicated by red arrowheads.

(d) Genome-wide differential analysis of dCas9 binding in K562 cells expressing HS2-specific sgRNA (sgHS2-rep1 and sgHS2-rep2) or non-targeting sgGal4. Data points for the sgRNA target regions and the predicted off-targets are shown as green and red, respectively. 
(e) Density maps are shown for DHS, ChIP-seq of H3K27ac, H3K4me1, H3K4me2, CTCF, and RNA-seq at the $\beta$-globin cluster (chr11: 5,222,500-5,323,700; hg19). The zoom-in view of the HS2 proximity region is shown on the top. Dashed lines denote the positions of sgRNAs.

(f) Expression of $\beta$-globin genes in $\mathrm{K} 562$ cells co-expressing enCRISPRi and target-specific sgRNAs at various positions within the $\beta$-globin cluster, control sgRNAs (sgCtrl, sgTAD1, sgTAD2, sgCTCF1 and sgCTCF2) or non-targeting sgGal4. mRNA expression relative to sgGal4 is shown as mean \pm SEM. The difference between control sgGal4 and other sgRNAs were analyzed by a two-way ANOVA. ${ }^{\star} P<0.05$, ${ }^{\star \star} P<0.01$, ${ }^{\star \star \star} P<0.001$, n.s. not significant. The differences between sgHS2 and other sgRNAs were analyzed by a two-way ANOVA. ${ }^{\#} P<0.05,{ }^{\# \#} P<0.01,{ }^{\# \#} P<0.001$.

\section{Figure 3. Locus-specific Epigenetic Reprogramming at the $\beta$-Globin Gene Cluster}

Density maps are shown for ChIP-seq of dCas9, active histone marks (H3K4me1, H3K4me2, and H3K27ac), repressive H3K9me3, GATA1, TAL1, and CTCF at the $\beta$-globin cluster (chr11: $5,222,500-5,323,700$; hg19) in K562 cells co-expressing non-targeting sgGal4 (control or $\mathrm{C}$ ) or sgHS2 with dCas9-KRAB (K), dCas9-LSD1 (L) or enCRISPRi (LK and KL). Regions showing increased or decreased ChIP-seq signals in enCRISPRi (LK) relative to control, dCas9-KRAB, dCas9-LSD1 or enCRISPRi (KL) (enCRISPRi - C, enCRISPRi - K, enCRISPRi - L, or enCRISPRi - KL) are depicted in green and red, respectively. Blue bars denote the sgRNAtargeted HS2 enhancer. Green bars denote the $\beta$-globin genes. Independent replicate experiments are shown as rep1 and rep2 in Figs. S1 and S2, respectively.

\section{Figure 4. Activation of TAL1 Oncogenic Super-Enhancer by enCRISPRa in Xenografts}

(a) Density maps are shown for ATAC-seq and H3K27ac ChIP-seq at the TAL1 locus (chr1:47,679,000-47,722,000; hg19) in Jurkat T-ALL cells. The annotated TAL1 oncogenic SE is shown as blue shaded lines. WT or Mut enhancer sequences are shown in zoom-in view. The positions of sgRNAs targeting WT or Mut allele and corresponding PAM sequences are shown as colored lines and boxes, respectively.

(b) Expression of TAL1 mRNA in Jurkat cells upon enCRISPRa-mediated activation of TAL1 oncogenic SE. Results are mean \pm SEM $(N=4$ independent experiments).

(c) Expression of TAL1 protein by Western blot. The quantified TAL1 expression is shown.

(d) Activation of TAL1 oncogenic SE promoted T-ALL growth in vitro. Cell proliferation was determined by PrestoBlue cell viability assays and shown as relative absorbance ( $y$-axis) after different days of culture (x-axis).

(e) Activation of TAL1 oncogenic SE promoted T-ALL growth in NSG mice xenografted with Jurkat cells transduced with control (sgGal4), sgMut2 or sgWT2, respectively. Bioluminescence intensity is shown for each mouse at 4 hours, 2- and 4-weeks posttransplantation.

(f) Quantification of bioluminescence intensity is shown for the xenografted NSG mice. Results are mean $\pm \operatorname{SEM}(N=5$ recipients per group). 
(g) Frequencies of leukemia cells in bone marrow (BM) and peripheral blood (PB) of xenografted NSG mice 4 weeks post-transplantation. Results are mean $\pm \operatorname{SEM}(N=5,5$, and 4 recipients for sgGal4, sgMut2 and sgWT2, respectively).

(h) Representative bloodsmear images of NSG mice 4 weeks after xenotransplantation of control (sgGal4), sgMut2 or sgWT2-transduced Jurkat cells. The inset images indicate the zoom-in view. The representative leukemia cells are indicated by arrowheads. Scale bars, $200 \mu \mathrm{m}$ and $20 \mu \mathrm{m}$ for full and insert images, respectively.

Results are mean \pm SEM and analyzed by a one-way or two-way ANOVA for multiple comparisons. ${ }^{\star} P<0.05,{ }^{\star \star} P<0.01$, ${ }^{\star \star \star} P<0.001$, n.s. not significant.

\section{Figure 5. Locus-Specific In Vivo Enhancer Perturbation}

(a) Schematic of site-specific KI of tetracycline-inducible dCas9-KRAB into the Col1a1 locus. Co-expression of dCas9-KRAB, sgRNA and MCP-KRAB leads to assembly of enCRISPRi complex in vivo.

(b) Validation of dCas9-KRAB and rtTA KI or WT alleles by genotyping PCR. C57BL/6 WT mouse and targeted $\mathrm{KH} 2-\mathrm{ESC}$ were used as controls. Two independent heterozygous (Het) and homozygous (Hom) KI mice were analyzed.

(c) Dox-inducible expression of dCas9-KRAB fusion protein was confirmed by Western blot in the targeted $\mathrm{KH} 2-\mathrm{ESC}$ and two independent dCas9-KRAB KI mice. $\beta$-tubulin was analyzed as the loading control.

(d) Schematic of in vivo perturbation of lineage-specific enhancers in dCas9-KRAB KI mice.

(e) In vivo enCRISPRi perturbation of Cebpa CREs revealed lineage-specific requirement of Cebpa enhancers during hematopoiesis. Waterfall plots are shown for target-specific sgRNAs (green and red dots) and non-targeting control sgRNAs (grey dots) by the mean normalized log2 fold changes in myeloid, $T$ or B cells 16-weeks post-BMT (T2) relative to pooled sgRNA-transduced HSPCs (T1) from two independent replicate screens ( $N=3$ recipient mice per screen). Density maps are shown for ATAC-seq and H3K27ac ChIP-seq at the Cebpa locus (chr7:35,877,000-35,951,000; mm9) in bone marrow HSC, granulocytes (GN), monocytes (Mono), B, CD4+ and CD8+ T cells, respectively. The annotated Cebpa promoter (P) and enhancers (E1 to E4) are indicated by green and blue shaded lines. Results from independent replicate screens and statistical analyses are shown in Fig. S6a,b.

(f) In vivo enCRISPRi perturbation of Spi1 CREs during hematopoiesis. Density maps are shown for ATAC-seq and H3K27ac ChIP-seq at the Spi1 locus (chr2:90,911,00090,957,000; mm9) in bone marrow HSC, GN, Mono, B, CD4+ and CD8+ T cells, respectively. The annotated Spi1 promoter $(P)$ and enhancer $(E)$ are indicated by green and blue shaded lines. Results from independent replicate screens and statistical analyses are shown in Fig. S6c,d.

Figure 6. Multiplexed Perturbation of Developmental Enhancers During Hematopoiesis 
(a) Schematic of in vivo multiplexed perturbation of developmentally regulated enhancers in dCas9-KRAB KI mice.

(b) In vivo perturbation of annotated CREs for five key hematopoietic TFs revealed the functional requirement of lineage-specific enhancers for HSC differentiation to one or multiple hematopoietic lineages. Waterfall plots are shown for target-specific sgRNAs (green and red dots) and non-targeting control sgRNAs (grey dots) by the mean normalized log2 fold changes in myeloid, T or B cells 16-weeks post-BMT (T2) relative to pooled sgRNAtransduced HSPCs (T1) from two independent replicate screens ( $N=15$ recipient mice per replicate screen). The annotated Runx1 promoters (P1 and P2) and enhancers (E1 to E3) are indicated by green and blue shaded lines. Results from independent replicate screens and statistical analyses are shown in Fig. S10.

(c) In vivo enCRISPRi of Runx1 CREs during hematopoiesis by single locus-based perturbation. Density maps are shown for ATAC-seq and H3K27ac ChIP-seq at the Runx1 locus (chr16:92,579,000-93,050,000; mm9) in bone marrow HSC, GN, Mono, B, CD4+ and CD8+ $\mathrm{T}$ cells, respectively. Results from independent replicate screens and statistical analyses are shown in Fig. S8a,b.

(d) In vivo enCRISPRi of Runx1 CREs during hematopoiesis by multiplexed perturbation. Results from independent replicate screens and statistical analyses are shown in Fig. S8c,d. 
bioRxiv preprint doi: https://doi.org/10.1101/761247; this version posted September 8, 2019. The copyright holder for this preprint (which was not certified by peer review) is the author/funder, who has granted bioRxiv a license to display the preprint in perpetuity. It is made available under aCC-BY-NC-ND 4.0 International license.

\section{Figure 1}

a

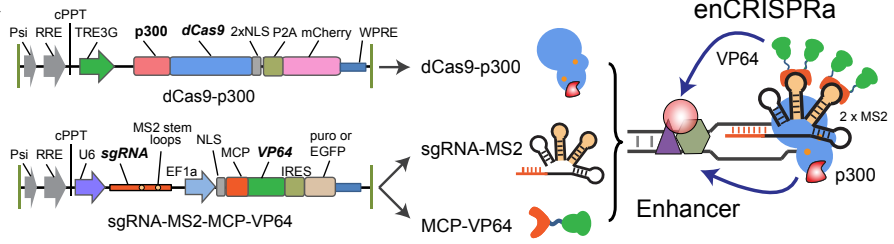

C
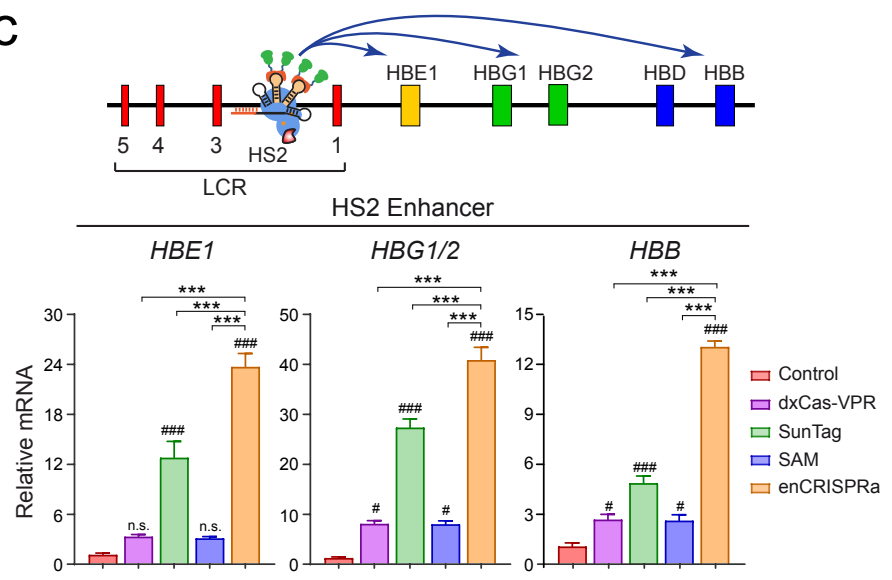

b

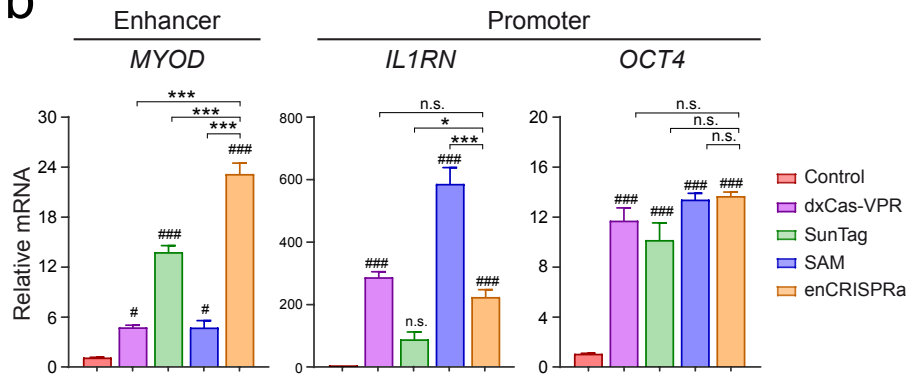

d

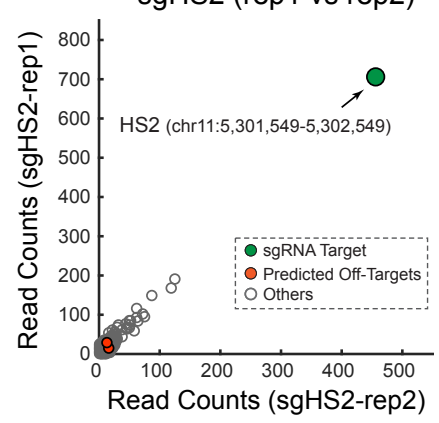

sgHS2 vs. sgGal4

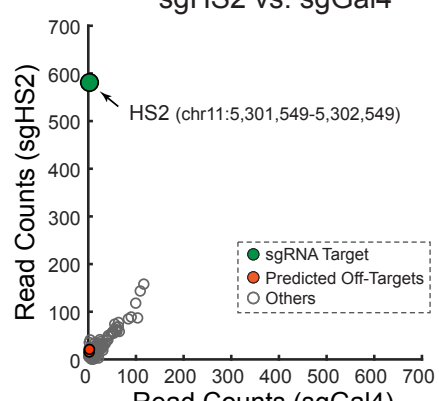

Read Counts (sgGal4) 
bioRxiv preprint doi: https://doi.org/10.1101/761247; this version posted September 8, 2019. The copyright holder for this preprint (which was not certified by peer review) is the author/funder, who has granted bioRxiv a license to display the preprint in perpetuity. It is made available under aCC-BY-NC-ND 4.0 International license.

\section{Figure 2}

a

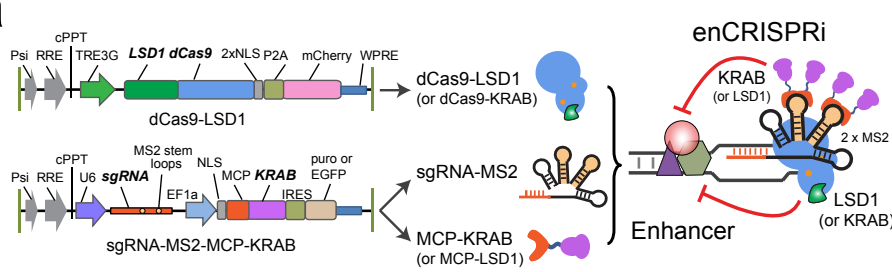

b
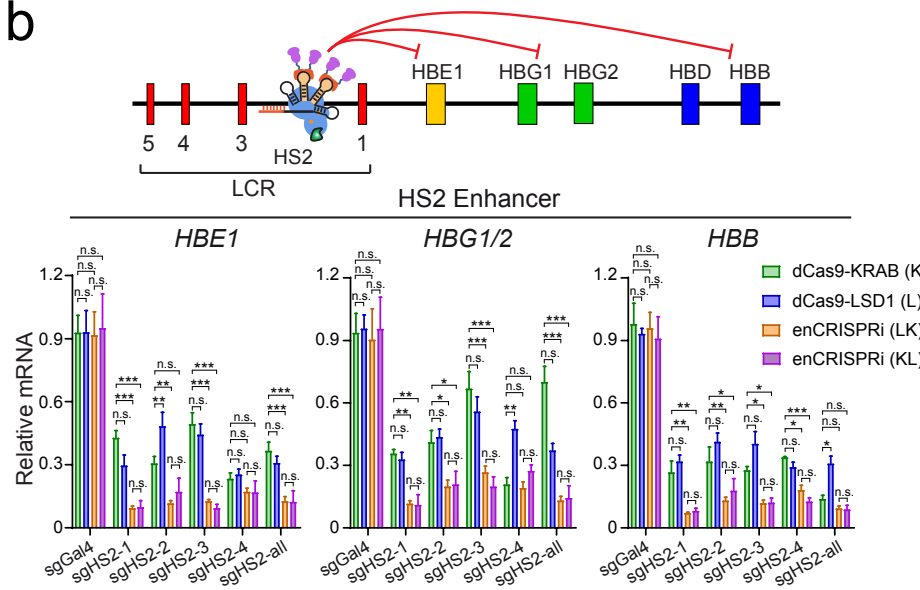

e
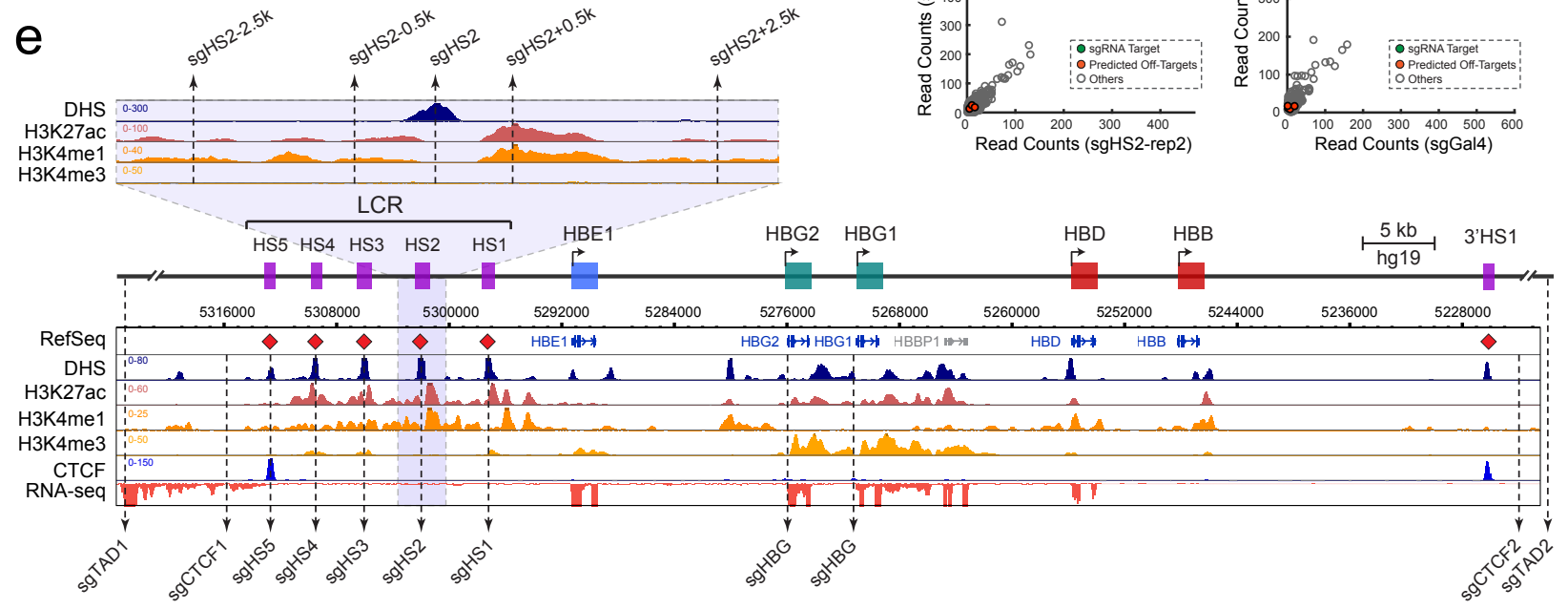

f
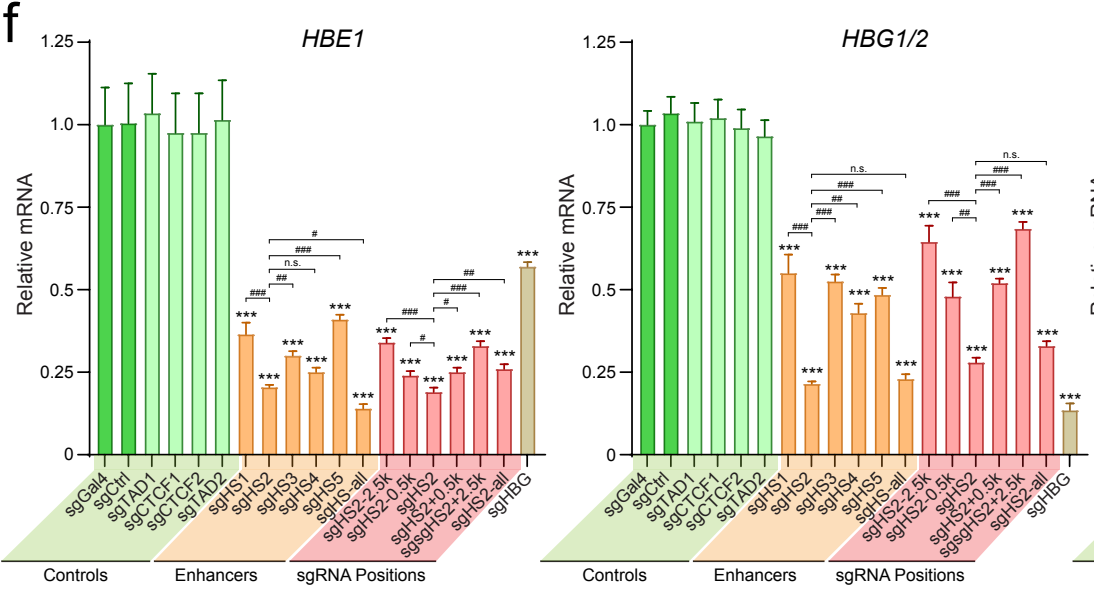
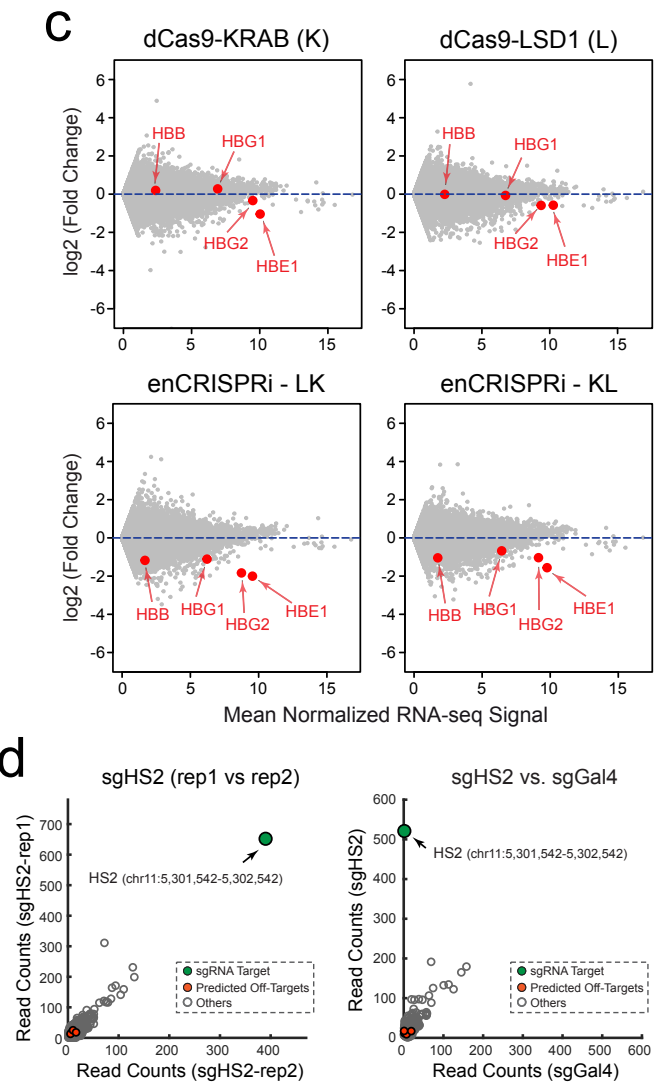

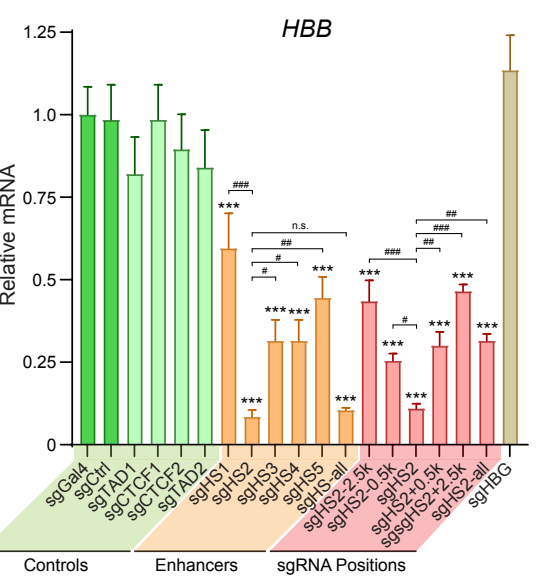


bioRxiv preprint doi: https://doi.org/10.1101/761247; this version posted September 8, 2019. The copyright holder for this preprint (which was not certified by peer review) is the author/funder, who has granted bioRxiv a license to display the preprint in perpetuity. It is made available under aCC-BY-NC-ND 4.0 International license.

\section{Figure 3}

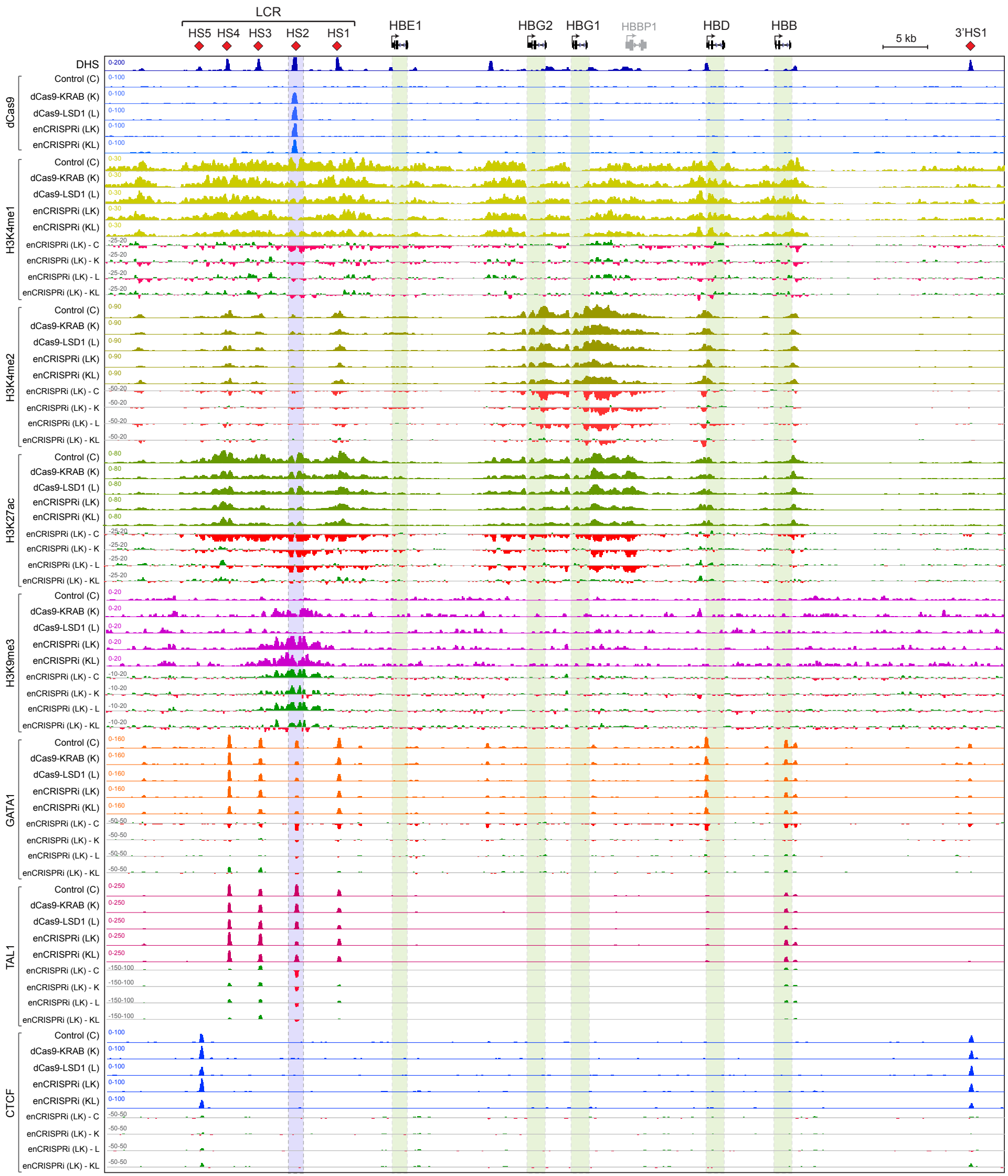


bioRxiv preprint doi: https://doi.org/10.1101/761247; this version posted September 8 2019. The copyright holder for this preprint (which was not certified by peer review) is the author/funder, who has granted bioRxiv a license to display the preprint in perpetuity. It is made available under aCC-BY-NC-ND 4.0 International license.

\section{Figure 4}

a

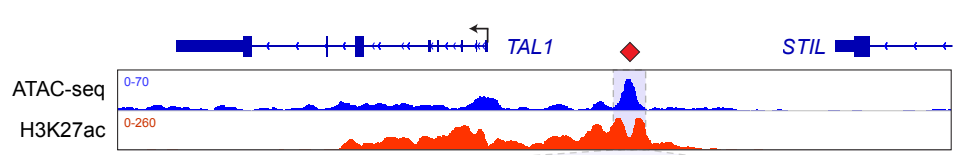

WT GATCCTGTGGTTGCCCCACCCCATTCCTATTACAGATAAACTGAGGGTCACAGAAAGACGTAACCCTACTTCCTGGCAG Mut GATCCTGTGGTTGCCCCACCCCATTCCTATTACAGATAAACTGAGGGTCACAGAAAGACGTAACCCTACTTCCTGGCAG

$$
\begin{aligned}
& \square \text { PAM } \\
& \equiv \text { sgRNAs }
\end{aligned}
$$

b

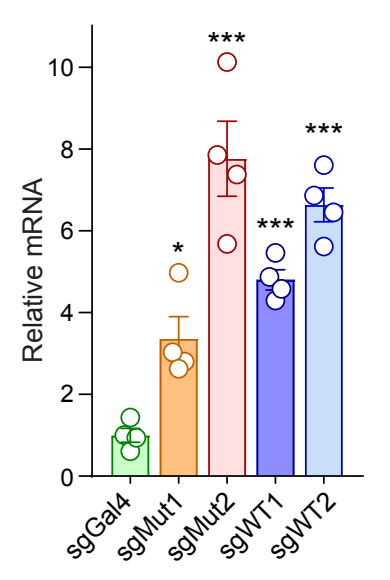

C
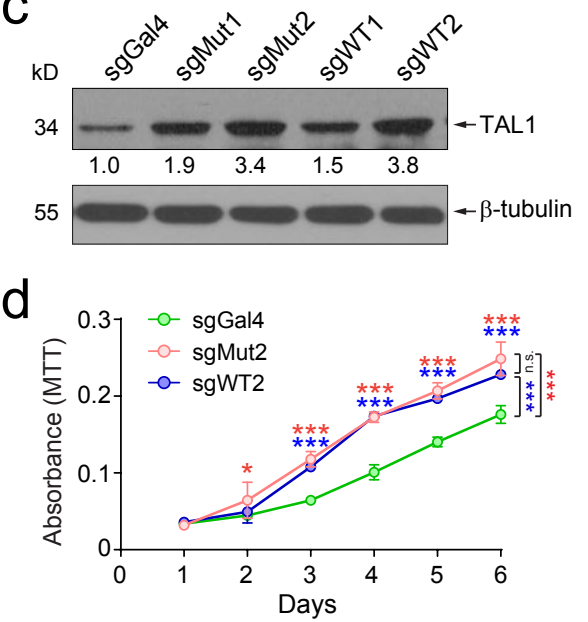

e

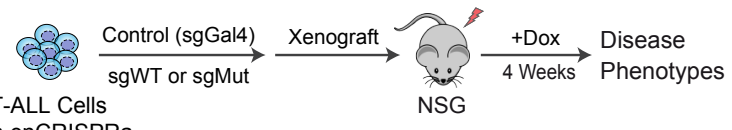
with enCRISPRa

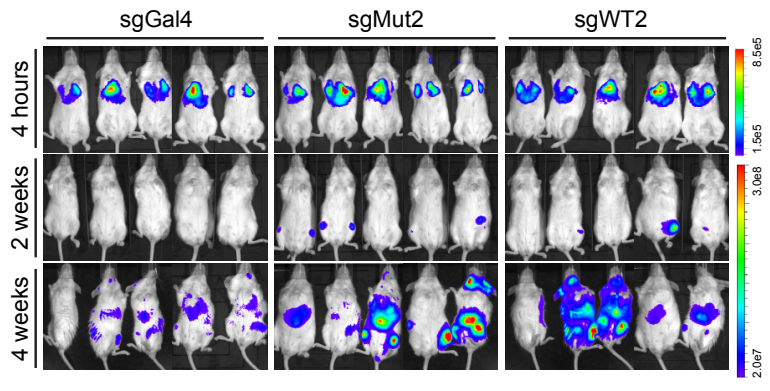

$f$
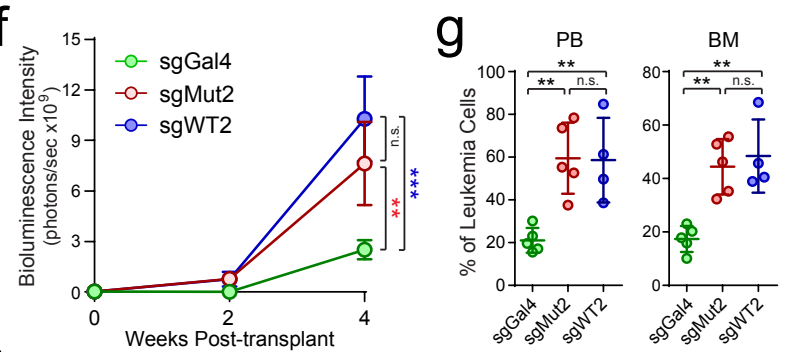

h

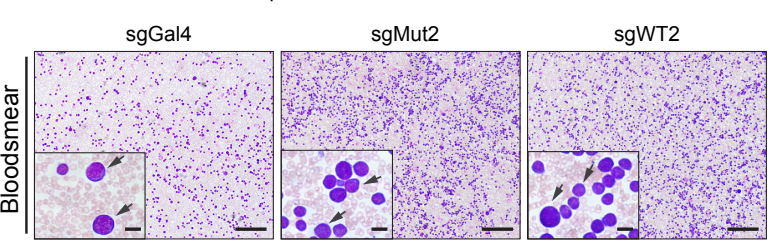


bioRxiv preprint doi: https://doi.org/10.1101/761247; this version posted September 8, 2019. The copyright holder for this preprint (which was not certified by peer review) is the author/funder, who has granted bioRxiv a license to display the preprint in perpetuity. It is made available under aCC-BY-NC-ND 4.0 International license.

\section{Figure 5}

a

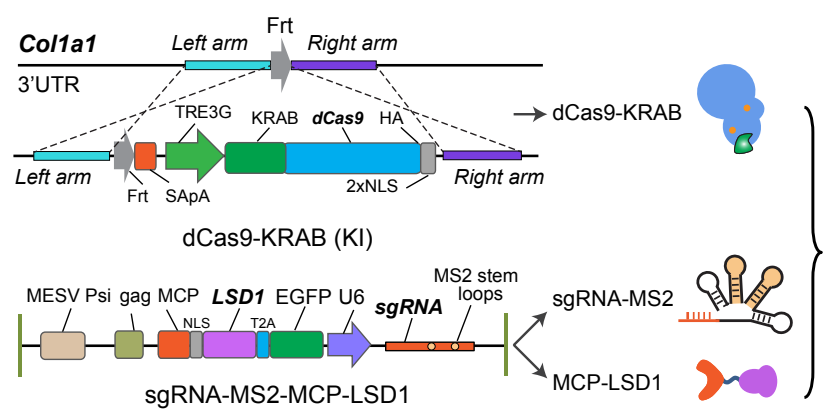

b

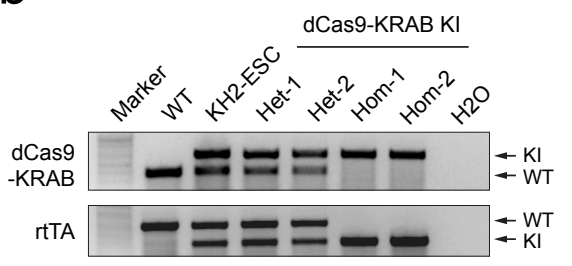

C

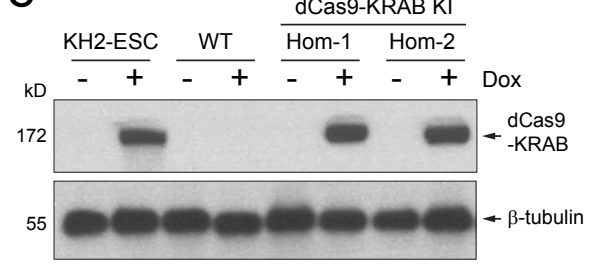

d

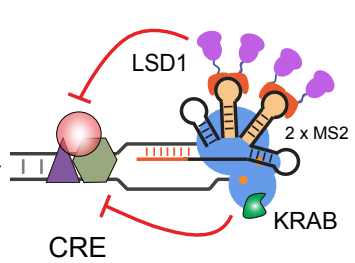

CRE
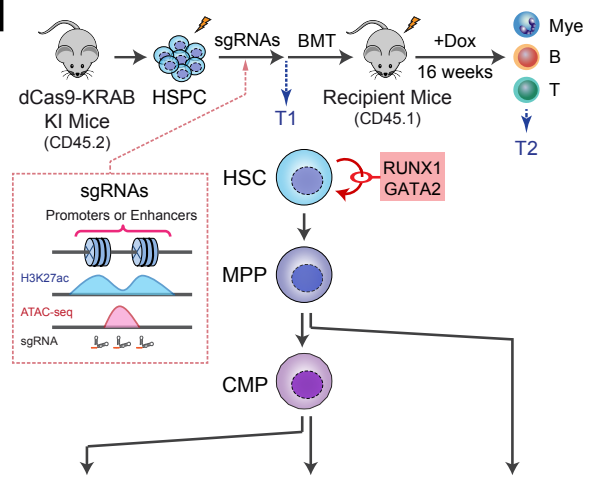

MEP

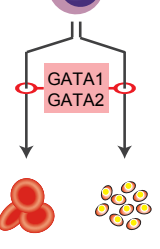

GMP

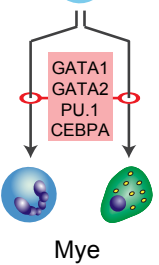

CLP

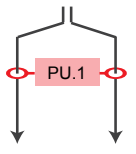

Ery Mye f
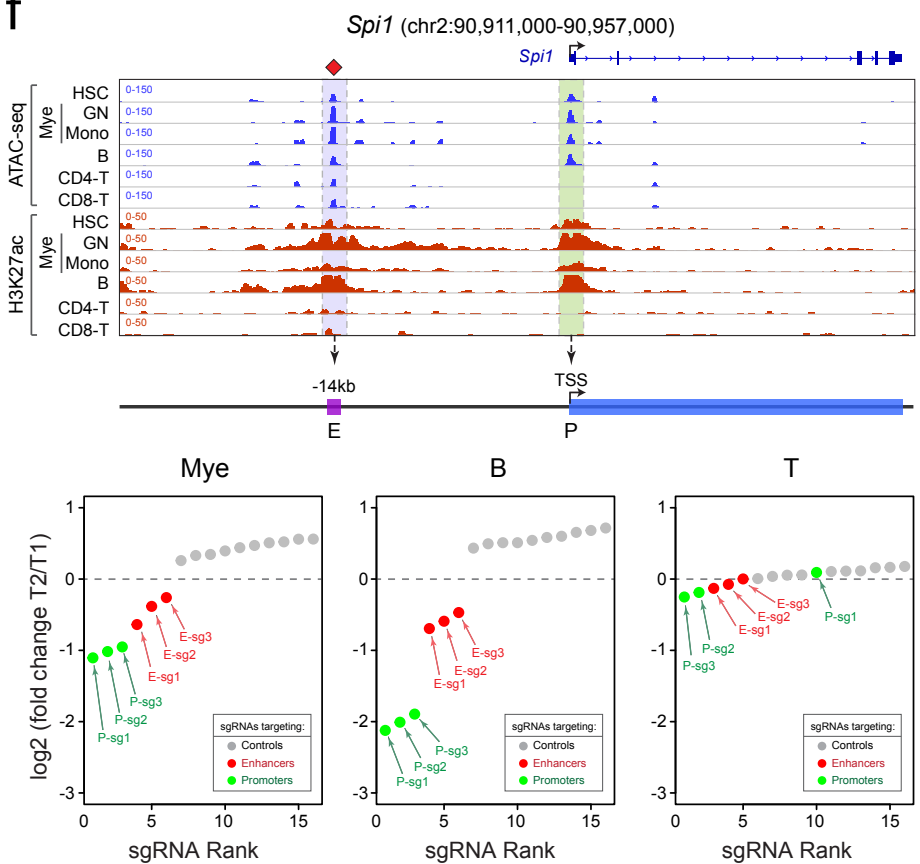

Mye

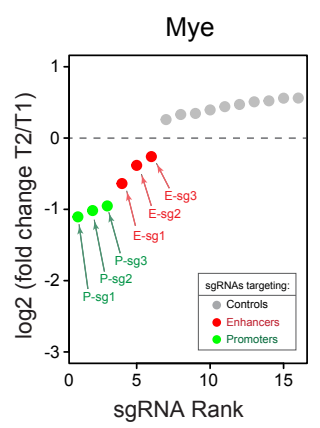

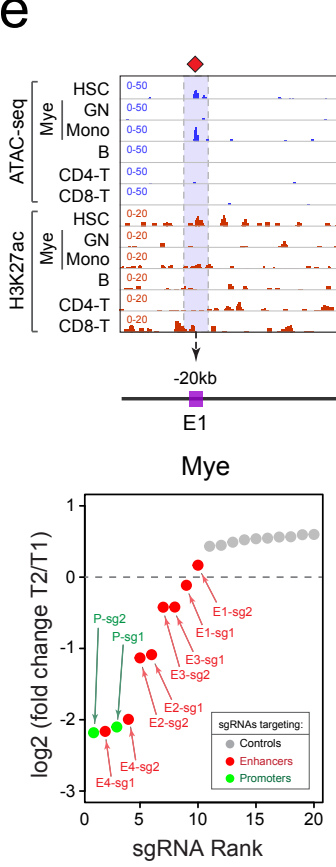

Cebpa (chr7:35,877,000-35,951,000)
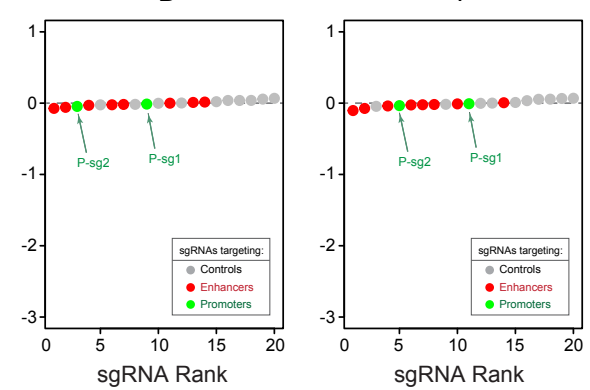
bioRxiv preprint doi: https://doi.org/10.1101/761247; this version posted September 8, 2019. The copyright holder for this preprint (which was not certified by peer review) is the author/funder, who has granted bioRxiv a license to display the preprint in perpetuity. It is made available under aCC-BY-NC-ND 4.0 International license.

\section{Figure 6}

a

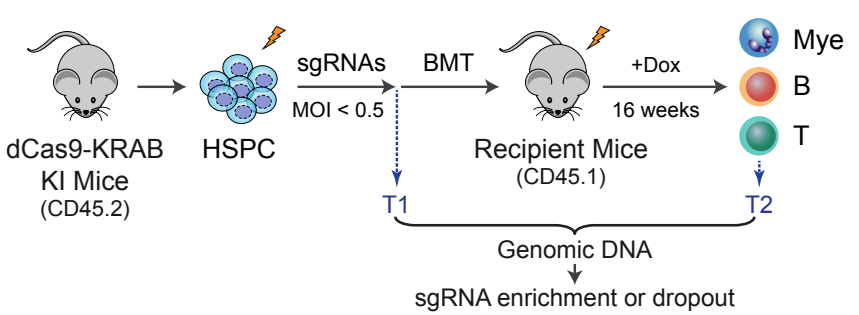

C
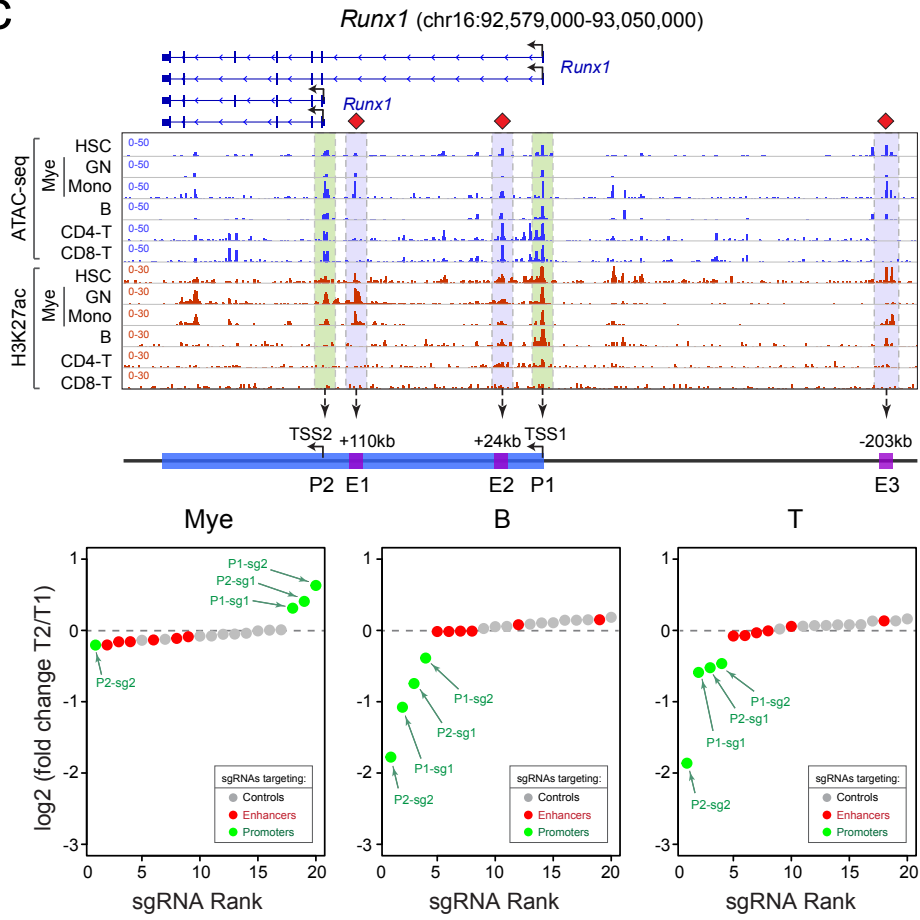

d
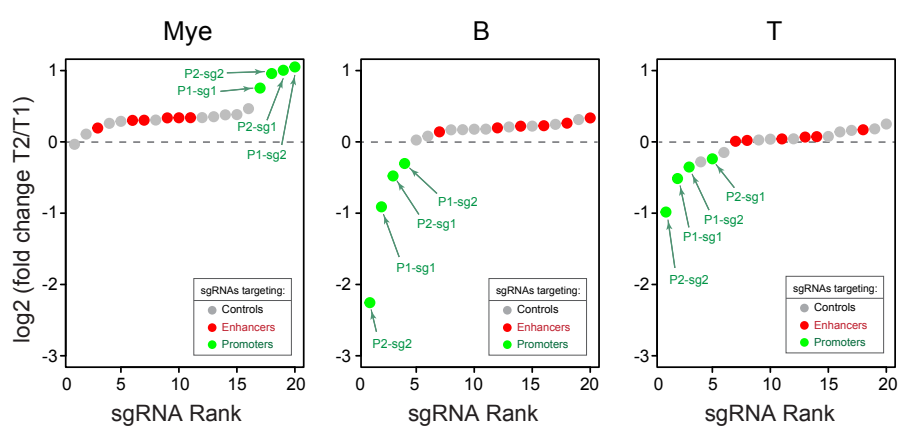

b

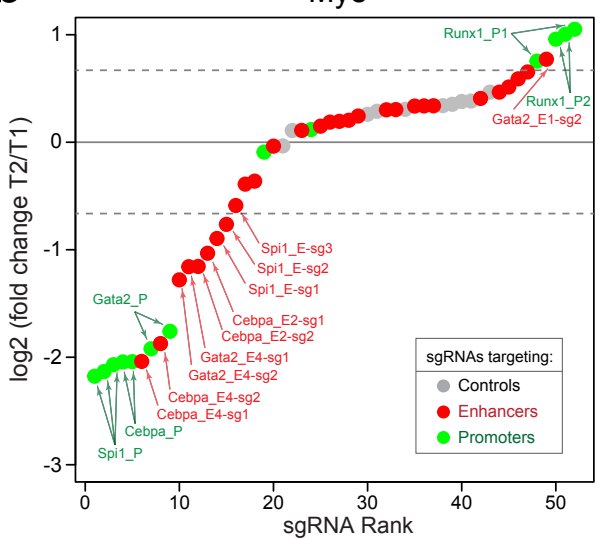

B

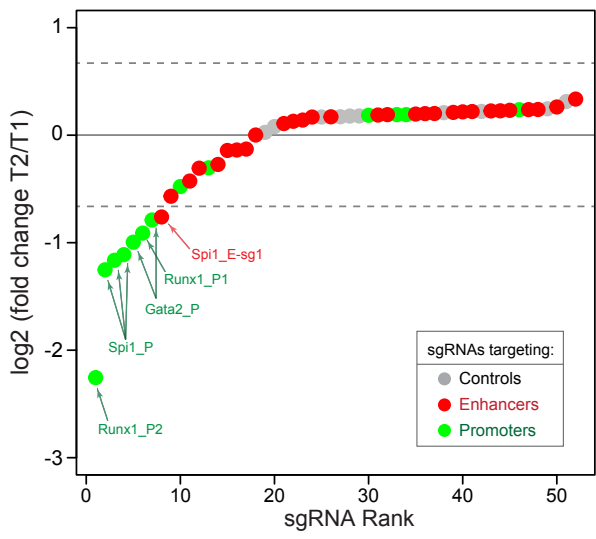

Top depleted: Runx1_P2-sg2 Spi1_P-sg1 Spi1_P-sg2 Spi1_P-sg3 Gata2_P-sg1 Gata2 P-sg2 Spi1 E-sg1 Spi1_E-sg2 Runx1_P2-sg1

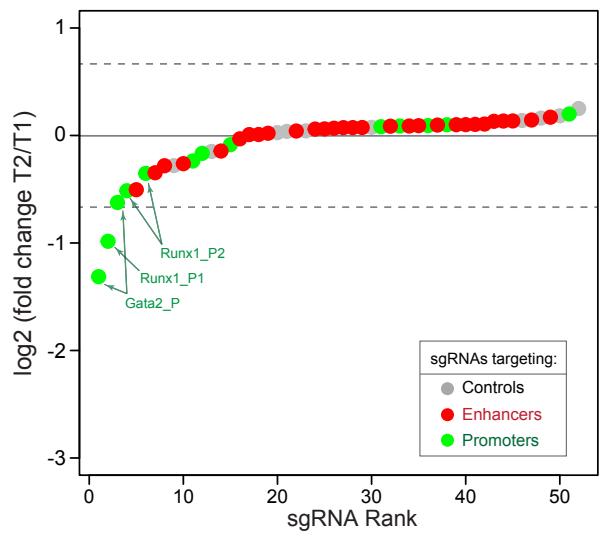

Top depleted: Gata2_P-sg1 Runx1_P2-sg2 Gata2_P-sg2 Runx1_P1-sg1 Gata2_E3-sg1 Gate- E1-sg1 Gata2_E1-sg1 Gata2_E4-sg2 